\title{
Portfolio Choice, Attention Allocation, and Price Comovement
}

\author{
Jordi Mondria* \\ University of Toronto ${ }^{\dagger}$
}

This draft: February 2010

\begin{abstract}
This paper models the attention allocation of portfolio investors. Investors choose the composition of their information subject to an information flow constraint. Given their expected investment strategy in the next period, which is to hold a diversified portfolio, in equilibrium investors choose to observe one linear combination of asset payoffs as a private signal. When investors use this private signal to update information about two assets, changes in one asset affect both asset prices and may lead to asset price comovement. The model also has implications for the transmission of volatility shocks between two assets.

Keywords: Rational Inattention, Asset Pricing, Portfolio Choice.
\end{abstract}

JEL Codes: D82, G12, G11.

${ }^{*}$ Economics Department. Max Gluskin House, Toronto, Ontario, M5S 3G7, Canada. Tel: 416-978-1494. Fax: 416-978-6713. E-mail: jordi.mondria@utoronto.ca.

†I am indebted to Hélène Rey, Chris Sims, Ricardo Reis and Wei Xiong for all their advice and guidance. I benefited from comments and discussions with Markus Brunnermeier, Carlos Carvalho, Vasco Cúrdia, Ettore Damiano, Wiola Dziuda, Rodrigo Guimaraes, Ken Kasa, Per Krusell, Pete Kyle, Katya Malinova, Andreas Park, Wolfgang Pesendorfer, Climent Quintana-Domeque, Antonella Tutino, Stijn Van Nieuwerburgh, Laura Veldkamp, Jonathan Vogel, Thomas Wu and all the seminar participants at Carlos III, CREI, IESE, Princeton, UAB, UBC, UCSC, U of Toronto, Econometric Society World Congress 2005 and Congress of the European Economic Association 2005. I would also like to thank Christian Hellwig (the editor) and two anonymous referees for their insightful and valuable comments. I gratefully acknowledge financial support from Fundación Rafael del Pino. All remaining errors are mine. 


\section{Introduction}

Many empirical findings in economics and finance have been explained by assuming a particular information structure. In recent years, there is a growing literature that lets agents choose the information structure, ${ }^{1}$ usually under some restrictions on its composition. In finance models, for example, investors are typically restricted to collect information about individual assets. However, in practice, information is also available about portfolios of assets. This paper studies a model in which investors can collect information about combinations of assets.

This paper presents a multiple asset, noisy rational expectations model with rationally inattentive agents that builds on Admati [1], Peng and Xiong [20] and Van Nieuwerburgh and Veldkamp $[25,26]$. The framework consists of two uncorrelated risky assets and a continuum of agents who face information processing constraints as in Sims $[22,23]$. Investors process information about the assets to reduce uncertainty about their portfolio. Investors process information in prices for free, have limited capacity to process additional information, and decide how to use this capacity. In particular, investors can choose between processing information about individual asset payoffs or linear combinations of asset payoffs. The information processing decision is formalized as choosing noisy signals about linear combinations of asset payoffs. After allocating their attention, investors incorporate the information from their private signals and from prices through Bayesian updating to form their posterior beliefs about the asset payoffs and then choose their optimal asset holdings.

This paper represents a step forward towards introducing rational inattention, introduced by Sims [22,23], in a general equilibrium finance model. Peng [19], Peng and Xiong [20] and Van Nieuwerburgh and Veldkamp [25,26] are the first attempts at introducing information capacity constraints into finance. In these models, if assets are assumed to be independent, then investors can process information only about individual assets because the ex-post variancecovariance matrix of the payoffs is constrained to be diagonal. In other words, in their approach, sources of uncertainty that are ex-ante independent remain independent ex-post.

In contrast, I solve for the optimal form of the ex-post variance-covariance matrix of the

\footnotetext{
${ }^{1}$ See Hellwig and Veldkamp [7], Luo [15], Mackowiak and Wiederholt [16,17], Peng [19], Peng and Xiong [20] and Van Nieuwerburgh and Veldkamp [25,26].
} 
payoffs, which is not initially constrained to be diagonal. Investors can get signals about any linear combination of asset payoffs. They are able to choose the composition of their information, i.e., investors decide if they want to observe a private signal about each asset payoff or about a linear combination of asset payoffs. Given their expected investment strategy in the next period, which is to hold a diversified portfolio, there is an equilibrium where all investors choose to observe a linear combination of asset payoffs as a private signal. This result is at the heart of the present paper. This result is consistent with the evidence provided by Hameed, Morck, Shen and Yeung [6]. They find that analysts follow assets that contain more valuable market and industry wide information, suggesting that investors use the information about some assets not only to trade in that asset, but also to value other assets. In other words, Hameed, Morck, Shen and Yeung [6] show that, in line with the central prediction of the present paper, investors choose to observe signals that are good predictors of many assets.

This model has implications for excess comovement in asset prices of seemingly unrelated assets. Excess comovement is defined as a high covariance of asset prices, relative to the covariance of their fundamentals. Excess comovement of asset prices affects the benefits of portfolio diversification and is, therefore, an important issue in economics and finance. ${ }^{2}$ This paper demonstrates that asset price comovement can be explained by rationally inattentive investors choosing to observe a linear combination of two uncorrelated asset payoffs. Intuitively, if there is good news about one asset, then investors observe a high realization of the private signal and they attribute part of the effect to one asset and the rest to the other asset. This leads to an increase in the price of both assets and, therefore, price comovement of uncorrelated assets. A numerical example with reasonable parameters for the U.S. stock market can generate an excess price correlation relative to fundamentals between $10.4 \%$ and $23.6 \%$ depending on the magnitude of the information processing capacity. These numbers are close to the average excess correlation of $25.5 \%$ between the index returns of all major industry groups in the U.S. stock market reported by Kallberg and Pasquariello [8].

\footnotetext{
${ }^{2}$ There is a large literature showing the existence of excess comovement of asset prices relative to the comovement of their fundamentals $(i)$ when there are announcements of common information content, (ii) when there are announcements of unrelated news, and ( $i i i)$ during non-announcement periods. See Pindyck and Rotemberg [21], Barberis, Shleifer and Wurgler [2] and Greenwood [5].
} 
Although other complementary channels are important for shedding light on asset price comovement, this paper studies a new mechanism: because investors choose to observe a linear combination of two uncorrelated asset payoffs as a private signal, changes in one asset are reflected in the information about the two assets and investors attribute part of the effect to one asset and the remaining to the other asset. Thus, provided investors have a limited capacity to process information, comovement between two uncorrelated assets arises even in the absence of correlated liquidity shocks (Calvo [3]), wealth effects (Kyle and Xiong [14]), direct or indirect macroeconomic links (King and Wadhwani [10], Kodres and Pritsker [13]), borrowing constraints (Yuan [29]) and endogenous information supply (Veldkamp [27]).

Empirically, it is difficult to distinguish between these different channels that potentially generate comovement. Nevertheless, this model has unique implications for the transmission of volatility shocks that distinguishes its mechanism from those of the alternative explanations. In my model, if there is an unexpected event that increases uncertainty about one asset, investors optimally allocate more attention to this asset, and therefore less attention to other assets. As a result, investors perceive these other assets to be riskier, and so my model predicts that the prices of these other stocks decline.

The evidence presented in Corwin and Coughenour [4] provides strong support for the implications of the model. They measure the attention allocated by a NYSE specialist to each asset, constructing a proxy for attention allocation based on the number of transactions and the absolute return during a given trading period. They find that when specialists increase the attention allocated to their most active stocks, there is an increase in the bid-ask spread of their remaining assigned stocks. This finding suggests that $(i)$ the information processing constraint is binding, even for professional investors, and (ii) an increase in the attention allocated to a group of assets generates an increase in the perceived volatility of the other assets assigned to the same specialist.

The remainder of the paper is organized as follows. Section 2 describes the model with special emphasis on rationally inattentive agents and information theory. Section 3 solves the investor's optimization problem. Section 4 examines price comovement between uncorrelated 
assets. Section 5 discusses the transmission of volatility shocks. Section 6 provides a numerical example with parameters chosen to reasonably match yearly asset price moments for the U.S. stock market. Section 7 concludes. The Appendix provides technical derivations and proofs.

\section{Model description}

There are two risky assets and a continuum of agents of measure one. Agents live three periods and are endowed with an initial wealth and a limited capacity to process information. In the first period, investors choose their private signal. In the second period, each investor decides on the optimal portfolio given the observation of a private signal and the price. In the last period, agents consume the payoff of their portfolio.

Each agent invests her initial endowment in three different assets: a risk free asset that pays $R_{f}$ units of the consumption good and two uncorrelated risky assets. The first risky asset pays

$\tilde{r}_{1} \sim N\left(\bar{r}_{1}, \sigma_{r 1}^{2}\right)$ units of the consumption good. The second risky asset pays $\tilde{r}_{2} \sim N\left(\bar{r}_{2}, \sigma_{r 2}^{2}\right)$ units of the consumption good. Let $\bar{R}$ and $\Sigma_{R}$ denote the mean vector and the diagonal variance-covariance matrix of the vector $\tilde{R}=\left(\tilde{r}_{1}, \tilde{r}_{2}\right)^{\prime}$. The numeraire in the market is the price of the bond and $\tilde{P}=\left(\tilde{p}_{1}, \tilde{p}_{2}\right)^{\prime}$ is the price vector of the risky assets. The net supply of the risky asset $j$ is given by the realization of a random variable $\tilde{z}_{j} \sim N\left(\bar{z}_{j}, \sigma_{z j}^{2}\right)$, where $\bar{z}_{j}>0$ for any risky asset $j$. Let $\bar{Z}$ and $\Sigma_{Z}$ denote the mean vector and the diagonal variance-covariance matrix of the vector of net supply $\tilde{Z}=\left(\tilde{z}_{1}, \tilde{z}_{2}\right)^{\prime}$. The vector of net supply, $\tilde{Z}$, is independent of the vector of asset payoffs, $\tilde{R}$. Asset supply randomness can be viewed as the result of some trade of a nonspeculative nature (liquidity traders) or some trade from agents lacking perfect knowledge of the market structure (irrational traders). This randomness is necessary in order to avoid perfect revelation of private information through the price.

\subsection{Information theory}

Investors face a constraint that limits the amount of information they can process. I will refer to this constraint as the information processing constraint. Intuitively, there is a large amount of information freely available relevant for decision making, but it takes time and 
mental attention for investors to incorporate this information into their decisions.

Following Sims [22,23], I use concepts from information theory to quantify the amount of information that a private signal contains about the asset payoffs. Information theory measures the rate of information flow as the rate of uncertainty reduction. Entropy is the measure of uncertainty used to calculate the information flow. This measure of uncertainty can be derived from four reasonable axioms, see Khinchin [9]. The entropy $H(X)$ of a random variable $X$ with a continuous probability density $p(x)$ is defined as

$$
H(X)=-E \ln [p(X)]=-\int p(x) \ln p(x) d x
$$

In the case where $X \sim N(\bar{X}, \Sigma)$ is an n-dimensional multivariate normal, its entropy is then

$$
H(X)=\frac{1}{2} \ln \left((2 \pi e)^{n}|\Sigma|\right)
$$

where $|\Sigma|$ is the determinant of $\Sigma$. Intuitively, for a Gaussian distributed variable, higher uncertainty is associated with higher variance.

According to information theory, the amount of information that a random variable contains about another random variable is measured as uncertainty reduction. This measure of uncertainty reduction is called mutual information. Consider two random variables $X$ and $Y$ with a joint density function $p(x, y)$ and marginal density functions $p(x)$ and $p(y)$. Mutual information $I(X ; Y)$ is defined as

$$
I(X ; Y)=\int p(x, y) \ln \frac{p(x, y)}{p(x) p(y)} d x d y=H(X)-H(X \mid Y)
$$

The amount of information that a random variable contains about another random variable is quantified as the difference between unconditional uncertainty and conditional uncertainty. Mutual information is invariant to any linear transformations of both random variables $X$ and $\mathrm{Y}$

$$
I(X ; Y)=I(a X+b ; c Y+d)
$$


This measure is independent of the scale of the underlying variables, unlike other measures of information such as the precision of the error in the private signal in Verrechia [28]. Given a limited information processing capacity, $\kappa,{ }^{3}$ which is the maximum rate at which agents can process information, the information processing constraint can be expressed as

$$
I(X ; Y)=H(X)-H(X \mid Y) \leq \kappa
$$

The information processing constraint limits the information flow by introducing an upper bound to the mutual information. Intuitively, this constraint restricts the amount of information contained in $Y$ about $X$.

\subsection{Investors' information processing constraint}

Investors want to obtain information about the risky assets in order to reduce the uncertainty of their optimal portfolio. However, agents have a limited capacity to process information about asset payoffs, which can be interpreted as limited time or mental attention. Investors face an information processing constraint as in Sims [22,23]. Agents optimally decide how much information they want to process about each asset given their limited information processing capacity and choose the form of the private signal through which they process this information. Agents are not allowed to process information about the net asset supply. In other words, the cost of processing information about the net asset supply is infinite.

I assume investor $i$ is constrained to choose a signal of the following form

$$
\tilde{Y}_{i}=C_{i} \tilde{R}+\tilde{\varepsilon}_{i} \text { where } \tilde{\varepsilon}_{i} \sim N\left(0, \Sigma_{i}\right)
$$

where $C_{i}$ is any $2 \times 2$ matrix, $\tilde{\varepsilon}_{i}$ is independent of $\tilde{R}$, and $\Sigma_{i}$ is the variance-covariance matrix of $\tilde{\varepsilon}_{i}$. The private signal provides information about linear combinations of asset payoffs. The precision of a signal is higher if more attention is allocated to that particular signal. The

\footnotetext{
${ }^{3}$ The information processing capacity is the infomation flow measure used in, among other things, characterizing modems or internet connections. An advantage of this measure of information is that there is no need to characterize the physical nature of the channel (wires, optical cables, human brain) through which the information is sent, it is enough to specify the capacity of the channel, $\kappa$.
} 
private signals are incorporated into the investor's beliefs through rational Bayesian updating. Investors optimally solve for the form of the conditional variance-covariance matrix of the payoffs by choosing $C_{i}$ and $\Sigma_{i}$ subject to the information processing constraint. Note that the conditional variance-covariance matrix of the payoffs is not constrained to be diagonal.

All investors have the same level of limited capacity to process information $\kappa$, which is assumed to be a strictly positive and finite parameter. For tractability reasons, they are assumed to process information about prices for free. ${ }^{4}$ Investors choose an optimal private signal to reduce the uncertainty about asset payoffs subject to the following information processing constraint

$$
H(\tilde{R})-H\left(\tilde{R} \mid \tilde{Y}_{i}\right) \leq \kappa
$$

which is written as in Peng [19] and Peng and Xiong [20]. ${ }^{5}$ Assuming Gaussian distributions for the asset payoffs and the private signals, the information processing constraint can be rewritten as

$$
\ln |\operatorname{Var}(\tilde{R})|-\ln \left|\operatorname{Var}\left(\tilde{R} \mid \tilde{Y}_{i}\right)\right| \leq 2 \kappa
$$

The information processing constraint restricts the information flow or, in other words, the amount of information about the asset payoffs contained in the private signal.

\subsection{Investors' objective function}

Investors, with a constant risk tolerance parameter $\rho$, maximize their expected certainty equivalent wealth

$$
E U_{i}=E\left\{-\ln E\left[\exp \left(-\frac{W_{i}^{\prime}}{\rho}\right) \mid \tilde{Y}_{i}, \tilde{P}\right]\right\}
$$

\footnotetext{
${ }^{4}$ See Mackowiak and Wiederholt [17] for a model where rationally inattentive agents do not perfectly observe prices.

${ }^{5}$ In Van Nieuwerburgh and Veldkamp [26], the information processing constraint restricts the mutual information between the asset payoffs, and prices and private information signals. All the results of the paper are robust to the information constraint used in Van Nieuwerburgh and Veldkamp [26] as shown in the additional appendix.
} 
where $W_{i}^{\prime}$ is the wealth of agent $i$ in the last period. ${ }^{6}$ The constant risk tolerance parameter $\rho$ is assumed to be strictly positive and finite. Following Van Nieuwerburgh and Veldkamp [25,26], I assume that investors have a preference for early resolution of uncertainty, as in Kreps and Porteus $[11,12] .{ }^{7}$ This is equivalent to maximizing a mean-variance objective function

$$
E U_{i}=\frac{1}{\rho} E\left(E\left[W_{i}^{\prime} \mid \tilde{Y}_{i}, \tilde{P}\right]-\frac{1}{2 \rho} \operatorname{Var}\left[W_{i}^{\prime} \mid \tilde{Y}_{i}, \tilde{P}\right]\right)
$$

Van Nieuwerburgh and Veldkamp [25] discuss this objective function in detail. One justification for these preferences is that there is an unmodeled intertemporal consumption choice. If investors had to choose their optimal consumption each period and they had a standard expected utility, then they would have a desire for early resolution of uncertainty as suggested by Spence and Zeckhauser [24]. Here the intertemporal consumption decision is not modeled because it unnecessarily complicates the model. Instead, I gain tractability while maintaining a preference for early resolution of uncertainty by employing Kreps and Porteus preferences.

Investors maximize their objective function subject to the following budget constraint

$$
W_{i}^{\prime}=W_{i 0} R_{f}+X_{i}^{\prime}\left(\tilde{R}-R_{f} \tilde{P}\right)
$$

where $W_{i 0}$ is the initial wealth of agent $i, X_{i}=\left(x_{i, 1}, x_{i, 2}\right)^{\prime}$ is the asset holdings vector of agent $i, \tilde{R}$ is the vector of risky asset payoffs and $\tilde{P}$ is the price vector of the risky assets. The market clearing conditions are given by $\int_{0}^{1} X_{i} d i=\tilde{Z}$.

\subsection{Timing}

In period one, investors choose $C_{i}$ and $\Sigma_{i}$ by maximizing the utility function in (3) or (4) subject to the information processing constraint in (2) and rational expectations about $X_{i}$. In period two, investors incorporate the information about asset payoffs from their optimally

\footnotetext{
${ }^{6}$ Ex post Gaussian uncertainty is typically optimal when the associated optimization problem is linearquadratic. Here Gaussian uncertainty is assumed for tractability.

${ }^{7} \mathrm{~A}$ preference for early resolution of uncertainty is expressed with a convex aggregator over the expected utility. I use the following convex transformation in order to obtain closed form solutions $f(x)=-\ln (-x)$ where $x=-E\left[\exp \left(-\frac{W_{i}^{\prime}}{\rho_{i}}\right) \mid \tilde{Y}_{i}, \tilde{P}\right]$.
} 
chosen private signal, $\tilde{Y}_{i}$, and the price, $\tilde{P}$, into their beliefs through Bayesian updating. Finally, given their posterior beliefs about asset payoffs, investors decide the optimal asset holdings, $X_{i}$, by maximizing the expected utility subject to the budget constraint in (5).

\section{Solving the model}

The model is solved using backward induction. First, given an arbitrary attention allocation, each agent decides the optimal asset holdings. Second, given the optimal risky asset demand as a function of each attention allocation, each agent chooses the optimal attention allocation.

\subsection{Optimal asset holdings}

In the second period, each agent chooses the optimal risky asset demand taking as given the private signal. After observing the private signal and the asset prices, investors derive their posterior beliefs about the payoffs in order to choose their optimal asset holdings

$$
X_{i}\left(\tilde{Y}_{i}, \tilde{P}\right)=\rho V_{i}^{-1} E\left[\tilde{R}-R_{f} \tilde{P} \mid \tilde{Y}_{i}, \tilde{P}\right]
$$

where

$$
V_{i}=\operatorname{Var}\left[\tilde{R} \mid \tilde{Y}_{i}, \tilde{P}\right]=\left(\Sigma_{R}^{-1}+\Pi \Sigma_{Z}^{-1} \Pi+C_{i}^{\prime} \Sigma_{i}^{-1} C_{i}\right)^{-1}
$$

The linear rational expectations equilibrium price is found by aggregating these asset demands and imposing the market clearing conditions.

Proposition 1 For a given signal choice by the investors, there exists a unique linear rational expectations equilibrium price vector.

A mean-variance objective function implies a linear demand for risky assets, which does not depend on wealth. If agents only faced the asset holdings decision given exogenous and independent private signals, then prices and holdings of both assets would be uncorrelated. 


\subsection{Attention allocation}

In the first period, each agent chooses the optimal attention allocation by maximizing her objective function taking into account the optimal asset demand. The following lemma expresses the first period objective function.

Lemma 1 The objective function for the attention allocation in the first period is given by

$$
E U_{i}=\frac{W_{i 0} R_{f}}{\rho}+\frac{1}{2}\left\{\operatorname{Tr}\left(V_{i}^{-1} Q-I\right)+\bar{R}^{e \prime} V_{i}^{-1} \bar{R}^{e}\right\}
$$

where $I$ is the identity matrix, $\operatorname{Tr}(\cdot)$ is the trace of a matrix, $V_{i}$ is given by (6) and $\bar{R}^{e}$ and $Q$ are respectively given by

$$
\begin{aligned}
& \bar{R}^{e}=E\left[E\left(\tilde{R} \mid \tilde{Y}_{i}, \tilde{P}\right)-R_{f} \tilde{P}\right]=\left(\rho \Sigma_{R}^{-1}+\rho \Pi \Sigma_{Z}^{-1} \Pi+\Pi\right)^{-1} \bar{Z} \\
& \quad \text { and } \\
& Q=\operatorname{Var}\left(\tilde{R}-R_{f} \tilde{P}\right)
\end{aligned}
$$

Investors choose the matrix of weights, $C_{i}$, and the variance-covariance matrix of the error term, $\Sigma_{i}$, in the private signal (1) by maximizing the utility function given by (7) subject to the information processing constraint given by (2). The following lemma shows that investors choose to observe one linear combination of asset payoffs as a private signal.

Lemma 2 In the first period, each investor allocates all of the limited information processing capacity to learn about one linear combination of asset payoffs.

Lemma 2 shows that for any matrix of weights, investors choose to learn about one linear combination of asset payoffs. Because investors are indifferent between observing a specific linear combination with either one or two private signals, I normalize the matrix of weights $C_{i}$ to be a $1 \times 2$ matrix such that $C_{i}=\left(c_{1 i}, c_{2 i}\right)$. This implies in particular that $\Sigma_{i}^{-1}$ is a scalar. Hence, investors receive a private signal of the following form $\tilde{Y}_{i}=c_{1 i} \tilde{r}_{1}+c_{2 i} \tilde{r}_{2}+\tilde{\varepsilon}_{i}$. The private signal contains more information about the first asset the higher the variance of 
the first term, $c_{1 i}^{2} \sigma_{r 1}^{2}$, and it contains more information about the second asset the higher the variance of the second term, $c_{2 i}^{2} \sigma_{r 2}^{2}$. Because investors only care about the relative weight that each asset has in the private signal, I also normalize the weight of the first asset in the private signal $c_{1 i}$ to 1 . The matrix of weights is then $C_{i}=\left(1, c_{2 i}\right)$, and the investor's decision problem is reduced to choosing the optimal relative weight of each asset in the private signal, $c_{2 i}$. The next proposition provides a parameter condition under which a unique linear symmetric rational expectations equilibrium exists and solves for the equilibrium values of $c_{2 i}$ and $\Sigma_{i}$. In the statement, $\bar{r}_{1}^{e+}, \bar{r}_{2}^{e+}$ and $Q_{12}^{+}$are functions of exogenous parameters and are defined in the appendix.

Proposition 2 A unique linear symmetric rational expectations equilibrium exists if and only if

$$
\bar{r}_{1}^{e+} \bar{r}_{2}^{e+}+Q_{12}^{+} \geq 0
$$

In this equilibrium, all investors allocate all their information processing capacity to learn about a unique linear combination of asset payoffs, $C=\left(1, c_{2}^{*}\right)$, with a weight in the second asset given by

$$
c_{2}^{*}=\frac{\left(\sigma_{r 2}^{2} \sigma_{z 2}^{2}+\sigma_{r 2}^{2} \bar{z}_{2}^{2}-\sigma_{r 1}^{2} \sigma_{z 1}^{2}-\sigma_{r 1}^{2} \bar{z}_{1}^{2}\right)+\sqrt{\left(\sigma_{r 2}^{2} \sigma_{z 2}^{2}+\sigma_{r 2}^{2} \bar{z}_{2}^{2}-\sigma_{r 1}^{2} \sigma_{z 1}^{2}-\sigma_{r 1}^{2} \bar{z}_{1}^{2}\right)^{2}+4 \sigma_{r 1}^{2} \sigma_{r 2}^{2} \bar{z}_{1}^{2} \bar{z}_{2}^{2}}}{2 \sigma_{r 2}^{2} \bar{z}_{1} \bar{z}_{2}}
$$

and a variance of the error term given by

$$
\Sigma^{*}=\frac{\sigma_{r 1}^{2}+c_{2}^{* 2} \sigma_{r 2}^{2}}{\left(e^{2 \kappa}-1\right)}
$$

Proposition 2 solves the attention allocation problem. There is a unique linear rational expectations equilibrium within the class of symmetric equilibria if and only if condition (9) is satisfied. This condition depends only on exogenous parameters. In equilibrium, $Q_{12}^{+}$is the covariance between the excess returns of both assets, $\bar{r}_{1}^{e+}$ and $\bar{r}_{2}^{e+}$ are the expected values of the excess returns. Consequently, condition (9) restricts the equilibrium covariance between excess returns of the two assets from being too negative. This parameter restriction is satisfied when 
the uncertainty in the economy is high relative to the level of risk tolerance and the amount of information that investors are able to process.

In this symmetric equilibrium, all investors choose to observe a linear combination of two asset payoffs as a private signal. Intuitively, given their expected investment strategy in the next period, which is to hold a diversified portfolio, the relevant information is about a linear combination of assets. ${ }^{8}$ One special feature of this equilibrium is that the weight of the second asset in the private signal is independent of the investors' information capacity, $\kappa$, and the investors' risk tolerance, $\rho$.

In this model, for some parameter values, there can be asymmetric equilibria. ${ }^{9}$ The next proposition shows that in any equilibrium, at least a positive fraction of investors chooses a private signal to learn about the two assets in the economy.

Proposition 3 In any equilibrium, a positive measure of investors chooses to learn about the two asset payoffs using only one linear combination as a private signal.

Proposition 3 implies, in particular, that an equilibrium where agents specialize in processing information about one asset or the other does not exist unlike in Van Nieuwerburgh and Veldkamp $[25,26]$. In their model, if risky assets are uncorrelated, a fraction of investors allocates all of their attention to the first asset and the remaining fraction of the agents pay attention only to the second asset. In their setup, increasing returns to information dominates diversification. In contrast, here, by introducing the more general form of private signals in (1), investors are able to exploit both increasing returns to information processing and diversification.

\footnotetext{
${ }^{8}$ In the additional appendix, I show that if investors are not able to process information from prices as in Peng and Xiong [20], then there exists a symmetric equilibrium where investors allocate all the information processing capacity to observe a private signal about the payoff of their expected portfolio. The optimal private signal in that case is given by $\tilde{Y}_{i}=\tilde{r}_{1}+\frac{\bar{z}_{2}}{\bar{z}_{1}} \tilde{r}_{2}+\tilde{\varepsilon}_{i}$, which is equivalent, as shown in the proof of Lemma 2 , to a signal about the payoff of the expected portfolio given by $\tilde{Y}_{i}^{*}=\bar{z}_{1} \tilde{Y}_{i}=\bar{z}_{1} \tilde{r}_{1}+\bar{z}_{2} \tilde{r}_{2}+\tilde{\varepsilon}_{i}^{*}$. However, if investors are able to process information from prices, the private signal is not exactly about the payoff of the expected portfolio. The reason is that, although investors care about the payoff of their expected portfolio, they choose a private signal taking into account the information they expect to learn from prices. Note that the expected portfolio is the expected supply, $E\left(X_{i}\right)=\bar{Z}$. The payoff of the expected portfolio is given by $\bar{Z}^{\prime} \tilde{R}=\bar{z}_{1} \tilde{r}_{1}+\bar{z}_{2} \tilde{r}_{2}$.

${ }^{9}$ In numerical simulations, for a wide range of parameters, I have been only able to construct asymmetric equilibria when $\bar{r}_{1}^{e+} \bar{r}_{2}^{e+}+Q_{12}^{+}<0$. See the additional appendix for more details on asymmetric equilibria.
} 


\subsection{Discussion}

The model presented has two formal differences relative to Van Nieuwerburgh and Veldkamp [26].

First, investors can choose a non-diagonal matrix of weights $C_{i}$ in the private signal in (1). In Van Nieuwerburgh and Veldkamp [25,26], as in Peng [19], Peng and Xiong [20], the matrix of weights in the private signal $C_{i}$ is the identity matrix. In the case in which the risky assets are ex-ante independent, they assume independence of uncertainty across assets expost, which means that investors collect separately information about each asset. In other words, they constrain the ex-post variance-covariance matrix of the payoffs to be diagonal, which means that independent sources of ex-ante uncertainty remain independent ex-post. Their assumption implies that an agent collects information one asset at a time, so that the only way to reduce uncertainty on a broad portfolio is to reduce variance on each asset, leaving the assets conditionally independent after the information collection. However, in this model, $C_{i}$ is optimally chosen by investors. In other words, investors optimally find the form of the conditional variance-covariance matrix of the payoffs, which is not initially constrained to be diagonal. For the case of two independent assets, according to Proposition 3, it is optimal at least for some investors to choose as a signal a weighted sum of asset payoffs instead of only receiving a signal about each asset. Also, according to Proposition 2, there exists a symmetric equilibrium in which all investors choose to observe a linear combination of the two assets in the economy as a private signal.

Second, asset prices do not appear in the information flow constraint. The information capacity constraint used in Van Nieuwerburgh and Veldkamp [26] is given by

$$
H(\tilde{R})-H\left(\tilde{R} \mid \tilde{P}, \tilde{Y}_{i}\right) \leq \kappa
$$

where $H(\tilde{R})$ is the entropy of the asset payoffs. In this information processing constraint, investors use information capacity when processing information from prices. However, in my model, investors do not use information capacity when getting information from prices as in Peng [19], Peng and Xiong [20]. Nevertheless, the difference in the information flow constraints 
plays no role in the different implications of the model with respect to Van Nieuwerburgh and Veldkamp [26]. ${ }^{10}$ In my model, there exists a symmetric equilibrium where all investors optimally devote all their attention to process information about the linear combination of asset payoffs given by (10) if the information processing constraint is that used by Van Nieuwerburgh and Veldkamp [26], given by (12).

\section{Implications for comovement of asset prices}

This section studies the implications of the linear symmetric equilibrium defined by (10) and (11) for stock price comovement of seemingly unrelated assets. In what follows, I describe the intuition behind the potential comovement. Investors choose to observe a linear combination of asset payoffs as a private signal. If they observe a high realization of the private signal, they attribute part of the effect to the first asset and the rest to the second asset. For a given of asset supply, a high realization of the private signal leads to an increase in the price of both assets and price comovement of seemingly unrelated assets. This is formalized in the following Proposition 4.

Proposition 4 Suppose condition (9) holds. Then in the linear symmetric equilibrium, for a given realization of asset supplies, $\tilde{Z}$, there is comovement of asset prices $\operatorname{Cov}\left(\tilde{P}_{1}, \tilde{P}_{2} \mid \tilde{Z}\right)>0$.

Although there is comovement of asset prices for a given asset supply according to Proposition 4, I obtain a similar unconditional result in Proposition 5 only under a parameter restriction.

Proposition 5 Suppose condition (9) holds and that

$$
\min \left\{\left(\rho^{2}-\sigma_{z 1}^{2} \sigma_{r 1}^{2}\right),\left(\rho^{2}-\sigma_{z 2}^{2} \sigma_{r 2}^{2}\right)\right\} \geq \frac{\sigma_{z 1}^{2} \sigma_{r 1}^{2} \sigma_{z 2}^{2} \sigma_{r 2}^{2}}{\rho^{2}}
$$

Then in the linear symmetric equilibrium, there is unconditional comovement of asset prices, $\operatorname{Cov}\left(\tilde{P}_{1}, \tilde{P}_{2}\right)>0$.

\footnotetext{
${ }^{10}$ See the additional appendix for details.
} 
According to the parameter restriction in Proposition 5, if investors' level of risk tolerance is high relative to the uncertainty in the economy, then there is stock price comovement. ${ }^{11}$ As argued above, an increase in the realization of one of the asset payoffs, increases both prices, yielding a positive covariance of asset prices. However, if this parameter restriction in Proposition 5 is violated, then an increase in the realization of one of the asset supplies may lead to a negative covariance of asset prices.

\section{Transmission of volatility shocks to asset prices}

This section studies whether the linear symmetric equilibrium defined by (10) and (11) offers an explanation for the transmission of volatility shocks in one asset to the prices of other seemingly unrelated assets. Intuitively, an unexpected event that raises the uncertainty of one asset $(i)$ may increase the attention allocated to this asset, $(i i)$ may raise the posterior uncertainty of the other uncorrelated asset through attention reallocation and thus (iii) may lead to a stock price decline of the other asset.

In the linear symmetric equilibrium, investors choose to receive a private signal of the following form $\tilde{Y}_{i}=\tilde{r}_{1}+c_{2}^{*} \tilde{r}_{2}+\tilde{\varepsilon}_{i}$. The private signal contains more information about the first asset the higher the variance of the first term, $\sigma_{r 1}^{2}$, and it contains more information about the second asset the higher the variance of the second term, $c_{2}^{* 2} \sigma_{r 2}^{2}$. Therefore, I measure the attention allocated to the first asset relative to the attention allocated to the second asset by $\frac{\sigma_{r 1}^{2}}{c_{2}^{* 2} \sigma_{r 2}^{2}}$. Proposition 6 formalizes the intuitive argument $(i)$ above by summarizing the impact of the assets' payoff and supply volatilities on investors' attention allocation.

Proposition 6 Suppose condition (9) holds. Then in the linear symmetric equilibrium, the relative attention allocated to asset $j=1,2$ increases with its payoff volatility, $\sigma_{r j}^{2}$, and supply volatility, $\sigma_{z j}^{2}$.

Investors allocate more attention to an asset the higher is its payoff or supply volatility and the lower is the payoff or supply volatility of the other asset. For expositional purposes,

\footnotetext{
${ }^{11}$ In the additional appendix, I show using numerical simulations that excess comovement of asset prices exists even in the presence of asymmetric equilibria.
} 
in what follows I focus on the second asset. The attention reallocation response to an increase in the uncertainty of the second asset payoff can be decomposed in two effects: a direct effect, which is given by an increase in the prior variance of the second asset, $\sigma_{r 2}^{2}$; and second, an indirect effect, which is given by an increase in the weight of the second asset in the private signal, $c_{2}^{*}$. Both effects generate an increase in the attention allocated to the second asset. In the direct effect, for a given weight of the second asset $c_{2}^{*}$, the private signal now provides more information about the second asset. The reason is that the private signal contains more information about the second asset the higher the variance of the second term, $c_{2}^{* 2} \sigma_{r 2}^{2}$. In the indirect effect, when there is an increase in prior variance of the second asset, $\sigma_{r 2}^{2}$, investors increase the weight of the second asset in the private signal, $\frac{\partial c_{2}^{*}}{\partial \sigma_{r 2}^{2}}>0$. This increases the information about the second asset since it increases the variance of the second term in the private signal, $c_{2}^{* 2} \sigma_{r 2}^{2}$.

Proposition 6 provides a testable implication for attention allocation: a variable that captures the information processed about a given industry should be higher in industries with a higher uncertainty about fundamentals. Another way to test the implication for attention allocation of Proposition 6 is by analyzing if an unexpected event that raises the uncertainty of one asset leads to a decrease in the information processed about other unrelated assets.

According to Proposition 6, an unexpected event that raises the uncertainty of the second asset decreases the attention allocated to the first asset. Proposition 7 formalizes the intuitive argument $(i i)$ above by showing that this decrease in the attention allocated to the first asset may lead to an increase in the posterior uncertainty of the first asset:

Proposition 7 Suppose condition (9) holds and that either

$$
\sigma_{z 2}^{2} \sigma_{r 2}^{2} \geq \rho^{2}\left(e^{2 \kappa}-1\right)
$$

or

$$
\sigma_{z 2}^{2} \sigma_{r 2}^{2} \geq \sigma_{z 1}^{2} \sigma_{r 1}^{2}
$$

Then in the linear symmetric equilibrium, an increase in the variance of the second asset leads 
to an increase in the posterior variance of the first asset.

The increase in the variance of the second asset spreads to the first asset through attention reallocation when one of the parameter restrictions in (13) or (14) is satisfied. If none of the parameter conditions is satisfied, it is possible that an increase in the variance in the second asset leads to a decrease in the conditional variance of the first asset due to the information content of prices.

To explain the intuition of this proposition, I decompose the attention reallocation effect in two pieces: first, an increase in the prior variance of the second asset, $\sigma_{r 2}^{2}$, and second, an increase in the weight of the second asset in the private signal, $c_{2}^{*}$. According to the first piece of the attention reallocation effect, if there is an increase in the variance of the second asset, then the signal, $\tilde{Y}_{i}$, provides less information about the first asset. This first piece of the attention reallocation effect, which abstracts from any change of the weights in the private signal, $c_{2}^{*}$, leads to an increase in the conditional variance of the first asset, $\operatorname{Var}\left[\tilde{r}_{1} \mid \tilde{Y}_{i}, \tilde{P}\right]$ without requiring any parameter restrictions.

The second piece of the attention reallocation effect is the change in the weights of the private signal. An increase in the variance of the second asset leads to an increase in the weight of the second asset in the private signal. Therefore, the signal provides less information about the first asset. The key to understand the possibility of a decrease in the posterior variance of the first asset is that a change in the weights of the private signal also changes the way investors learn from prices. If neither (13) nor (14) are satisfied, it is possible that the reduction in information processed about the first asset through the private signal is dominated by the increase in information processed about this first asset by asset prices, leading to a decrease in the posterior variance of the first asset.

Proposition 7 provides a testable implication for the perceived volatility of investors: if (13) or (14) hold, an increase in the attention allocated to one asset leads to an increase in the perceived volatility of other unrelated assets.

According to Proposition 7 an unexpected event that raises the uncertainty of the second asset raises the posterior uncertainty of the first asset through attention reallocation under 
the parameter condition (14). Proposition 8 formalizes the intuitive argument (iii) above by showing that under the same parameter restriction, such unexpected event also leads to a stock price decline of the first asset.

Proposition 8 If conditions (9) and (14) hold, then in the linear symmetric equilibrium, an increase in the variance of the second asset leads to a decrease in the expected price of the first asset.

If the parameter restriction (14) is satisfied and there is an increase in the uncertainty of the second asset, then there is an increase in the attention allocated to this asset, and the concurrent decrease in the attention allocated to the first asset. This leads to an increase of the posterior variance of the first asset and an increase in its risk premium. Hence, the expected excess return of the first asset increases and its expected price falls. If the parameter restriction (14) is not satisfied, it is possible that an increase in the variance of one asset leads to an increase in the expected price of the other asset.

\section{Numerical analysis}

This section conducts a numerical example with parameters chosen to reasonably match yearly asset price moments for the U.S. stock market. I take my parameter values from Van Nieuwerburgh and Veldkamp [26]. Each asset payoff has a standard deviation of prior beliefs $\sigma_{r 1}=\sigma_{r 2}=15 \%$, which reflects historical levels of payoff volatility, and an expected payoff $\bar{r}_{1}=\bar{r}_{2}=1$. The mean of the asset payoffs does not affect second moments. Each asset has an expected net supply $\bar{z}_{1}=\bar{z}_{2}=100$ and a considerably high standard deviation $\sigma_{z 1}=\sigma_{z 2}=10$ for prices not to reveal too much information. The coefficient of risk tolerance is $\rho=2$. The return of the riskless asset is $2 \%$, so that $R_{f}=1.02 .{ }^{12}$

Panel A in figure 1 shows that, as long as the information processing capacity, $\kappa$, is higher than zero, stock prices are correlated even though fundamentals are uncorrelated. In this figure and for these particular parameter values, the higher the information capacity, the higher is

\footnotetext{
${ }^{12}$ These parameter values satisfy the conditions in Propositions 2, 5, 7 and 8.
} 
the level of correlation. Intuitively, if the information capacity is zero, no information is processed and stocks are uncorrelated. However, if there is a positive information processing capacity, then because the parameter restriction in Proposition 5 is satisfied, there is asset price comovement. An information processing capacity $\kappa=0.4$ implies an asset price correlation of $21 \%$. This asset price correlation predicted by the model is close to the average excess correlation of $25.5 \%$ between the index returns of all major industry groups in the U.S. stock market reported by Kallberg and Pasquariello [8]. ${ }^{13}$

Panel B in figure 1 shows that if information capacity is held constant at $\kappa=0.4$ and the standard deviation of the prior beliefs in the second asset is increased to $\sigma_{r 2}=17 \%$, then the expected price of the second asset decreases by $11.2 \%$ and the expected price of the first asset decreases by $2.26 \%$.

\section{Conclusion}

This paper presents a rational expectations model of asset prices with information processing constraints and explains asset price comovement and transmission of volatility shocks between seemingly unrelated assets.

The model represents a step forward towards the introduction of rational inattention into a general equilibrium finance model. Investors choose the composition of their information, i.e., investors can process information about individual assets or linear combination of assets. Unlike previous papers, where investors collect information about individual assets, investors optimally decide the form of the conditional variance-covariance matrix of the asset payoffs, which is not initially constrained to be diagonal. However, several assumptions should be relaxed in order to have a general equilibrium model with rationally inattentive agents. The model assumes that investors have Gaussian uncertainty about the random variable of interest, in this case asset payoffs, ex-ante and ex-post. Sims [23] presents a methodology to find the nature of the ex-post uncertainty about the random variable of interest. Another assumption of

\footnotetext{
${ }^{13}$ Pasquariello and Vega [18] reported the correlation matrix of the earnings of all major industry groups in the U.S. stock market and noted that the fundamentals of many industries in the U.S. stock market are uncorrelated.
} 
the model is that rationally inattentive agents are able to freely observe prices, which means that investors have an unbounded capacity to process information about prices. They are also unable to process information about the random asset supply, so the cost of processing information about the noisy asset supply is infinite. A potential extension of the model is to relax these two assumptions by having investors who observe prices with a measurement error. Then, the noisy asset supply assumption can be dropped since prices are unobservable. In this case, I conjecture that investors will not process information about prices or payoffs individually, but the variable of interest for investors will be the asset excess returns. Hence, investors, when deciding their asset holdings, will not pay attention to the price, but they will process information about the asset excess returns.

\section{Appendix}

\subsection{Proof of Proposition 1}

Following Admati [1], one can show that there exists a unique linear rational expectations equilibrium price vector. Define $\Pi$ as

$$
\Pi=\int_{0}^{1} \rho C_{i}^{\prime} \Sigma_{i}^{-1} C_{i} d i
$$

The price vector is given by

$$
\tilde{P}=A_{0}+A_{1} \tilde{R}-A_{2} \tilde{Z}, \text { with } A_{2} \text { nonsingular }
$$

where

$$
\begin{aligned}
& A_{0}=\frac{\rho}{R_{f}}\left(\rho \Sigma_{R}^{-1}+\rho \Pi \Sigma_{Z}^{-1} \Pi+\Pi\right)^{-1}\left(\Sigma_{R}^{-1} \bar{R}+\Pi \Sigma_{Z}^{-1} \bar{Z}\right) \\
& A_{1}=\frac{1}{R_{f}}\left(\rho \Sigma_{R}^{-1}+\rho \Pi \Sigma_{Z}^{-1} \Pi+\Pi\right)^{-1}\left(\Pi+\rho \Pi \Sigma_{Z}^{-1} \Pi\right) \\
& A_{2}=\frac{1}{R_{f}}\left(\rho \Sigma_{R}^{-1}+\rho \Pi \Sigma_{Z}^{-1} \Pi+\Pi\right)^{-1}\left(I+\rho \Pi \Sigma_{Z}^{-1}\right)
\end{aligned}
$$

\subsection{Proof of Lemma 1}

The objective function in the first period is given by (4) where

$$
E\left[W_{i}^{\prime} \mid \tilde{Y}_{i}, \tilde{P}\right]=W_{i 0} R_{f}+\rho E\left[\tilde{R}-R_{f} \tilde{P} \mid \tilde{Y}_{i}, \tilde{P}\right]^{\prime} \operatorname{Var}\left[\tilde{R} \mid \tilde{Y}_{i}, \tilde{P}\right]^{-1} E\left[\tilde{R}-R_{f} \tilde{P} \mid \tilde{Y}_{i}, \tilde{P}\right]
$$

and

$$
\operatorname{Var}\left[W_{i}^{\prime} \mid \tilde{Y}_{i}, \tilde{P}\right]=\rho^{2} E\left[\tilde{R}-R_{f} \tilde{P} \mid \tilde{Y}_{i}, \tilde{P}\right]^{\prime} \operatorname{Var}\left[\tilde{R} \mid \tilde{Y}_{i}, \tilde{P}\right]^{-1} E\left[\tilde{R}-R_{f} \tilde{P} \mid \tilde{Y}_{i}, \tilde{P}\right]
$$


Define $V_{E R}$ as the variance-covariance matrix of the conditional expected excess returns

$$
V_{E R}=\operatorname{Var}\left(E\left[\tilde{R}-R_{f} \tilde{P} \mid \tilde{Y}_{i}, \tilde{P}\right]\right)
$$

By standard results from statistics, if $X$ and $Y$ are arbitrary random variables for which the necessary expectations and variances exist, then $\operatorname{Var}(Y)=E[\operatorname{Var}(Y \mid X)]+\operatorname{Var}(E[Y \mid X])$. Using this result, the variance-covariance matrix of the conditional expected excess returns is given by

$$
\begin{aligned}
V_{E R} & =\operatorname{Var}\left(E\left[\tilde{R}-R_{f} \tilde{P} \mid \tilde{Y}_{i}, \tilde{P}\right]\right) \\
& =\operatorname{Var}\left(\tilde{R}-R_{f} \tilde{P}\right)-\operatorname{Var}\left(\tilde{R} \mid \tilde{Y}_{i}, \tilde{P}\right) \\
& =\Sigma_{R}+R_{f}^{2} A_{1} \Sigma_{R} A_{1}^{\prime}+R_{f}^{2} A_{2} \Sigma_{Z} A_{2}^{\prime}-R_{f} A_{1} \Sigma_{R}-R_{f} \Sigma_{R} A_{1}^{\prime}-V_{i}
\end{aligned}
$$

where $V_{i}$ is given by (6). By standard results from statistics, if $x=\left(x_{1}, x_{2}, \ldots, x_{n}\right)^{\prime} \sim N(\mu, V)$ and $q=x^{\prime} A x$, then the expected value of $q$ is $E(q)=\operatorname{tr}[A V]+\mu^{\prime} A \mu$. Using this result, the objective function in (4) is given by

$$
E U_{i}=\frac{W_{i 0} R_{f}}{\rho}+\frac{1}{2}\left\{\operatorname{Tr}\left(V_{i}^{-1} Q-I\right)+\bar{R}^{e \prime} V_{i}^{-1} \bar{R}^{e}\right\}
$$

where $I$ is the identity matrix, $\bar{R}^{e}$ is given by (8) and $Q$ is given by

$$
Q=\operatorname{Var}\left(\tilde{R}-R_{f} \tilde{P}\right)=\Sigma_{R}+R_{f}^{2} A_{1} \Sigma_{R} A_{1}^{\prime}+R_{f}^{2} A_{2} \Sigma_{Z} A_{2}^{\prime}-R_{f} A_{1} \Sigma_{R}-R_{f} \Sigma_{R} A_{1}^{\prime}
$$

\subsection{Proof of Lemma 2}

I will follow three steps to show that each investor allocates all of the limited information processing capacity to learn about one linear combination of asset payoffs. First, I will show that a normalization of the variance-covariance matrix of the error term in the private signal, $\Sigma_{i}$, is required. Second, I will show that a normalization of the matrix of weights, $C_{i}$, is required. Finally, I will solve the optimization problem of an infinitesimal investor.

Step1: Normalization of $\Sigma_{i}$

Given that the variance-covariance matrix of the error term in the private signal, $\Sigma_{i}$, can be decomposed as follows

$$
\Sigma_{i}=P_{i} \Lambda_{i} P_{i}^{\prime}
$$

where $\Lambda_{i}$ is a diagonal matrix and $P^{\prime}=P^{-1}$, I will show that for every non-diagonal $\Sigma_{i}$, an equilibrium with a private signal $\tilde{Y}_{i}$ is indistinguishable from an equilibrium with a private signal $\tilde{Y}_{i}^{*}$ given by

$$
\tilde{Y}_{i}^{*}=P_{i}^{-1} \tilde{Y}_{i}=P_{i}^{-1} C_{i} \tilde{R}+P_{i}^{-1} \tilde{\varepsilon}_{i}=C_{i}^{*} \tilde{R}+\tilde{\varepsilon}_{i}^{*}
$$

where $\operatorname{Var}\left(\tilde{\varepsilon}_{i}^{*}\right)=\Lambda_{i}$ is a diagonal matrix.

First, I show that the new private signal satisfies the information capacity constraint. Mutual Information is invariant to any invertible linear transformation of the random variables, which implies

$$
I(X ; Y)=I\left(X ; P^{-1} Y\right)=H(X)-H(X \mid Y) \leq \kappa
$$

Second, in order to prove that an equilibrium with a private signal $\tilde{Y}_{i}$ is indistinguishable from an 
equilibrium with a private signal $\tilde{Y}_{i}^{*}$, there is enough to show that $C_{i}^{\prime} \Sigma_{i}^{-1} C_{i}=C_{i}^{\prime}\left(P_{i} \Lambda_{i} P_{i}^{\prime}\right)^{-1} C_{i}=$ $C_{i}^{* \prime} \Lambda_{i}^{-1} C_{i}^{*}$. More details are provided in Admati [1].

Therefore, for a given matrix of weights $C_{i}$, I let investors choose a normalized diagonal variancecovariance matrix of the error term in the private signal

$$
\Sigma_{i}=\left(\begin{array}{cc}
\sigma_{i 1}^{2} & 0 \\
0 & \sigma_{i 2}^{2}
\end{array}\right)
$$

Step 2: Normalization of $C_{i}$

I will show that for every matrix of weights,$C_{i}$, and diagonal variance-covariance matrix of the error term, $\Sigma_{i}$, an equilibrium with a private signal $\tilde{Y}_{i}$ is indistinguishable from an equilibrium with a private signal $\tilde{Y}_{i}^{*}$ given by

$$
\tilde{Y}_{i}^{*}=\Gamma \tilde{Y}_{i}=\Gamma C_{i} \tilde{R}+\Gamma \tilde{\varepsilon}_{i}=C_{i}^{*} \tilde{R}+\tilde{\varepsilon}_{i}^{*}
$$

where $\Gamma$ is a diagonal non-singular matrix and $\operatorname{Var}\left(\tilde{\varepsilon}_{i}^{*}\right)$ is still a diagonal matrix.

First, I show that the new private signal satisfies the normalization of the variance-covariance matrix of the error term in the private signal, $\Sigma_{i}$, from step 1 . Because $\Gamma$ is a diagonal matrix, then the variance-covariance matrix of the new error term, $\tilde{\varepsilon}_{i}^{*}=\Gamma \tilde{\varepsilon}_{i}$, is still diagonal and given by $\Gamma \Sigma_{i} \Gamma$. Second, as in step 1 of this proof, the new private signal satisfies the information capacity constraint. Finally, in order to prove that an equilibrium with a private signal $\tilde{Y}_{i}$ is indistinguishable from an equilibrium with a private signal $\tilde{Y}_{i}^{*}$, there is enough to show that $C_{i}^{\prime} \Sigma_{i}^{-1} C_{i}=C_{i}^{\prime} \Gamma\left(\Gamma \Sigma_{i} \Gamma\right)^{-1} \Gamma C_{i}=C_{i}^{* \prime}\left(\Gamma \Sigma_{i} \Gamma\right)^{-1} C_{i}^{*}$. More details are provided in Admati [1].

Therefore, I normalize the first column of $C_{i}$ to be a column of ones such that the matrix of weights is given by

$$
C_{i}=\left(\begin{array}{cc}
1 & c_{i 12} \\
1 & c_{i 22}
\end{array}\right)
$$

\section{Step 3: Optimization problem of an infinitesimal investor.}

According to steps 1 and 2, for a given normalized matrix of weights, $C_{i}$, given by (19), I can let investors choose a normalized diagonal variance-covariance matrix of the error term in the private signal given by (18). Investors maximize the objective function given by (7) that can be expressed as

$$
\begin{aligned}
& \max _{\sigma_{i 1}^{-2}, \sigma_{i 2}^{-2}}\left(\left(\left(\bar{r}_{1}^{e}\right)^{2}+Q_{11}\right)+\left(\left(\bar{r}_{2}^{e}\right)^{2}+Q_{22}\right) c_{i 12}^{2}+2\left(\bar{r}_{1}^{e} \bar{r}_{2}^{e}+Q_{12}\right) c_{i 12}\right) \sigma_{i 1}^{-2}+ \\
& +\left(\left(\left(\bar{r}_{1}^{e}\right)^{2}+Q_{11}\right)+\left(\left(\bar{r}_{2}^{e}\right)^{2}+Q_{22}\right) c_{i 22}^{2}+2\left(\bar{r}_{1}^{e} \bar{r}_{2}^{e}+Q_{12}\right) c_{i 22}\right) \sigma_{i 2}^{-2}+\Omega
\end{aligned}
$$

where

$$
\begin{aligned}
\Omega=\frac{W_{i 0} R_{f}}{\rho}+ & \frac{1}{2}\left[\left(\frac{1}{\sigma_{r 1}^{2}}+\frac{\pi_{11}^{2}}{\sigma_{z 1}^{2}}+\frac{\pi_{12}^{2}}{\sigma_{z 2}^{2}}\right)\left(\left(\bar{r}_{1}^{e}\right)^{2}+Q_{11}\right)+2\left(\frac{\pi_{11} \pi_{12}}{\sigma_{z 1}^{2}}+\frac{\pi_{12} \pi_{22}}{\sigma_{z 2}^{2}}\right)\left(\bar{r}_{1}^{e} \bar{r}_{2}^{e}+Q_{12}\right)+\right. \\
& \left.+\left(\frac{1}{\sigma_{r 2}^{2}}+\frac{\pi_{12}^{2}}{\sigma_{z 1}^{2}}+\frac{\pi_{22}^{2}}{\sigma_{z 2}^{2}}\right)\left(\left(\bar{r}_{2}^{e}\right)^{2}+Q_{22}\right)-1\right] \text { is a constant term }
\end{aligned}
$$

subject to the information constraint given by (2) that can be expressed as

$$
\left(\sigma_{r 1}^{2}+c_{i 12}^{2} \sigma_{r 2}^{2}\right) \sigma_{i 1}^{-2}+\left(\sigma_{r 1}^{2}+c_{i 22}^{2} \sigma_{r 2}^{2}\right) \sigma_{i 2}^{-2}+\sigma_{r 1}^{2} \sigma_{r 2}^{2}\left(c_{i 22}-c_{i 12}\right)^{2} \sigma_{i 1}^{-2} \sigma_{i 2}^{-2}=\left(e^{2 \kappa}-1\right)
$$

where $\pi_{11}, \pi_{12}$ and $\pi_{22}$ are each of the elements in the matrix $\Pi$ given by (15), $\bar{r}_{1}^{e}$ and $\bar{r}_{2}^{e}$ are each of 
the elements of the expected excess returns vector $\bar{R}^{e}$ given by (8) and $Q_{11}, Q_{12}$ and $Q_{22}$ are each of the elements in the matrix $Q$ given by (17). The investor when optimizing takes as given $\bar{r}_{1}^{e}, \bar{r}_{2}^{e}, \pi_{11}$, $\pi_{12}, \pi_{22}, Q_{11}, Q_{12}$ and $Q_{22}$. The optimization problem can be rewritten as

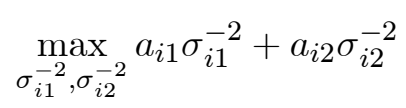

subject to

$$
b_{i 1} \sigma_{i 1}^{-2}+b_{i 2} \sigma_{i 2}^{-2}+b_{i 3} \sigma_{i 1}^{-2} \sigma_{i 2}^{-2}=b_{i 4} ; \sigma_{i 1}^{-2} \geq 0 ; \sigma_{i 2}^{-2} \geq 0
$$

where $a_{i 1}, a_{i 2}, b_{i 1}, b_{i 2}$ and $b_{i 4}$ are strictly positive constants and $b_{i 3} \geq 0$. If the first constraint, which is the information constraint, is introduced into the objective function, the maximization problem becomes

$$
\max _{\sigma_{i 1}^{-2}} a_{i 1} \sigma_{i 1}^{-2}+a_{i 2}\left(\frac{b_{i 4}-b_{i 1} \sigma_{i 1}^{-2}}{b_{i 2}+b_{i 3} \sigma_{i 1}^{-2}}\right) \text { where } \sigma_{i 1}^{-2} \in\left[0, \frac{b_{i 4}}{b_{i 1}}\right]
$$

The second order condition is given by

$$
\frac{\partial^{2}}{\partial\left(\sigma_{i 1}^{-2}\right)^{2}}=\frac{2 a_{i 2}\left(b_{i 1} b_{i 2}+b_{i 3} b_{i 4}\right) b_{i 3}}{\left(b_{i 2}+b_{i 3} \sigma_{i 1}^{-2}\right)^{3}} \geq 0
$$

If $b_{i 3}>0$, then the objective function is a strictly convex function over a compact set, $\sigma_{i 1}^{-2} \in\left[0, \frac{b_{i 4}}{b_{i 1}}\right]$. Thus, the solution to the optimization problem is a corner solution and investors allocate all their attention to one linear combination of asset payoffs. If $b_{i 3}=0$, then $c_{i 22}=c_{i 12}$, the objective function is independent of the choice variable and there is a continuum of solutions. Investors are indifferent between any two private signals with identical weights of the asset payoffs. This implies that investors are indifferent with any allocation of limited information capacity between the two signals. Therefore, investors choose to learn about one linear combination of asset payoffs, but they can do so by observing one or two private signals. In this case, when $c_{i 22}=c_{i 12}$, I normalize the matrix of weights $C_{i}$ to be a $1 \times 2$ matrix, which implies that investors are restricted to observe one private signal.

\subsection{Proof of Proposition 2}

I will show that there is a unique linear symmetric rational expectations equilibrium if and only if condition (9) holds. The proof proceeds in five steps. First, I will solve the optimization problem of an infinitesimal investor. In step 2, I will characterize all potential symmetric equilibria and show that there are four candidates. In step 3, I will eliminate three of these symmetric candidates. In step 4, I will show that there is a unique linear symmetric rational expectations equilibrium if $\bar{r}_{1}^{e+} \bar{r}_{2}^{e+}+Q_{12}^{+} \geq 0$ and solve for the equilibrium values of $c_{2 i}$ and $\Sigma_{i}$. Finally, in step 5, I will conclude that there exists a linear symmetric rational expectations equilibrium only if $\bar{r}_{1}^{e+} \bar{r}_{2}^{e+}+Q_{12}^{+} \geq 0$.

Step 1: Solve the infinitesimal agent's optimization problem.

Investors maximize the objective function given by (7) subject to the information capacity constraint

$$
\Sigma_{i}^{-1}=\frac{\left(e^{2 \kappa}-1\right)}{\sigma_{r 1}^{2}+c_{2 i}^{2} \sigma_{r 2}^{2}}
$$

Following the proof of Lemma 2, I normalize the matrix of weights to be $C_{i}=\left(1, c_{2 i}\right)$. Substituting the information constraint given by (21) into the objective function given by (7), the optimization 
problem becomes

$$
\max _{c_{2 i}} \frac{\left[\left(\left(\bar{r}_{1}^{e}\right)^{2}+Q_{11}\right)+\left(\left(\bar{r}_{2}^{e}\right)^{2}+Q_{22}\right) c_{2 i}^{2}+2\left(\bar{r}_{1}^{e} \bar{r}_{2}^{e}+Q_{12}\right) c_{2 i}\right]\left(e^{2 \kappa}-1\right)}{\sigma_{r 1}^{2}+c_{2 i}^{2} \sigma_{r 2}^{2}}+\Omega
$$

where $\Omega$ is given by (20), $\bar{r}_{1}^{e}$ and $\bar{r}_{2}^{e}$ are given by (8) and $Q_{11}, Q_{12}$ and $Q_{22}$ are given by (17). Infinitesimal investors have no effect on prices and take as given $\Omega, \bar{r}_{1}^{e}, \bar{r}_{2}^{e}, Q_{11}, Q_{12}$ and $Q_{22}$ when optimizing. The first order condition is given by

$$
\begin{gathered}
{\left[-\sigma_{r 2}^{2}\left(\bar{r}_{1}^{e} \bar{r}_{2}^{e}+Q_{12}\right) c_{2 i}^{2}-\left(\sigma_{r 2}^{2}\left(\left(\bar{r}_{1}^{e}\right)^{2}+Q_{11}\right)-\sigma_{r 1}^{2}\left(\left(\bar{r}_{2}^{e}\right)^{2}+Q_{22}\right)\right) c_{2 i}+\right.} \\
\left.+\sigma_{r 1}^{2}\left(\bar{r}_{1}^{e} \bar{r}_{2}^{e}+Q_{12}\right)\right] \frac{2}{\left(\sigma_{r 1}^{2}+c_{2 i}^{2} \sigma_{r 2}^{2}\right)^{2}}=0
\end{gathered}
$$

Step 1.1: Best response when $\left(\bar{r}_{1}^{e} \bar{r}_{2}^{e}+Q_{12}\right) \neq 0$

I will show that the best response of an infinitesimal investor when $\left(\bar{r}_{1}^{e} \bar{r}_{2}^{e}+Q_{12}\right) \neq 0$ is given by

$$
c_{2 i}^{*}=\frac{\left[\sigma_{r 1}^{2}\left(\left(\bar{r}_{2}^{e}\right)^{2}+Q_{22}\right)-\sigma_{r 2}^{2}\left(\left(\bar{r}_{1}^{e}\right)^{2}+Q_{11}\right)\right]+\sqrt{\left[\sigma_{r 1}^{2}\left(\left(\bar{r}_{2}^{e}\right)^{2}+Q_{22}\right)-\sigma_{r 2}^{2}\left(\left(\bar{r}_{1}^{e}\right)^{2}+Q_{11}\right)\right]^{2}+4 \sigma_{r 1}^{2} \sigma_{r 2}^{2}\left(\bar{r}_{1}^{e} \bar{r}_{2}^{e}+Q_{12}\right)^{2}}}{2\left[\sigma_{r 2}^{2}\left(\bar{r}_{1}^{e} \bar{r}_{2}^{e}+Q_{12}\right)\right]}
$$

If $\left(\bar{r}_{1}^{e} \bar{r}_{2}^{e}+Q_{12}\right) \neq 0$, the first order condition (23) has two interior solutions. One is a local maximum and one is a local minimum. The local maximum is given by (24). This is the reaction function where investors take as given the aggregate variables of the economy. The relevant term in the second order condition evaluated at $c_{2 i}=c_{2 i}^{*}$ is given by

$$
-2 \sigma_{r 2}^{2}\left(\bar{r}_{1}^{e} \bar{r}_{2}^{e}+Q_{12}\right) c_{2 i}^{*}-\left[\left(\sigma_{r 2}^{2}\left(\left(\bar{r}_{1}^{e}\right)^{2}+Q_{11}\right)-\sigma_{r 1}^{2}\left(\left(\bar{r}_{2}^{e}\right)^{2}+Q_{22}\right)\right]<0\right.
$$

The other interior solution to the first order condition is always a local minimum. The local maximum $c_{2 i}^{*}$ given by (24) is a global maximum because the second order condition at this point is strictly negative and the objective function is not maximized by setting $c_{2 i}= \pm \infty$.

Step 1.2: Best response when $\left(\bar{r}_{1}^{e} \bar{r}_{2}^{e}+Q_{12}\right)=0$.

I will show that the best response of an infinitesimal investor when $\left(\bar{r}_{1}^{e} \bar{r}_{2}^{e}+Q_{12}\right)=0$ is given by either $c_{2 i}^{*}= \pm \infty$ or $c_{2 i}^{*}=0$ or the objective function is independent of $c_{2 i}$ and any $c_{2 i}$ is a best response.

If $\left(\bar{r}_{1}^{e} \bar{r}_{2}^{e}+Q_{12}\right)=0$, the first order condition (23) is given by

$$
\left[-\left(\sigma_{r 2}^{2}\left(\left(\bar{r}_{1}^{e}\right)^{2}+Q_{11}\right)-\sigma_{r 1}^{2}\left(\left(\bar{r}_{2}^{e}\right)^{2}+Q_{22}\right)\right) c_{2 i}\right] \frac{2}{\left(\sigma_{r 1}^{2}+c_{2 i}^{2} \sigma_{r 2}^{2}\right)^{2}}=0
$$

If $\left(\bar{r}_{1}^{e} \bar{r}_{2}^{e}+Q_{12}\right)=0$ and $\left(\sigma_{r 2}^{2}\left(\left(\bar{r}_{1}^{e}\right)^{2}+Q_{11}\right)>\sigma_{r 1}^{2}\left(\left(\bar{r}_{2}^{e}\right)^{2}+Q_{22}\right)\right)$, then $c_{2 i}=0$ is a global maximum.

If $\left(\bar{r}_{1}^{e} \bar{r}_{2}^{e}+Q_{12}\right)=0$ and $\left(\sigma_{r 2}^{2}\left(\left(\bar{r}_{1}^{e}\right)^{2}+Q_{11}\right)<\sigma_{r 1}^{2}\left(\left(\bar{r}_{2}^{e}\right)^{2}+Q_{22}\right)\right)$, then $c_{2 i}= \pm \infty$ is a global maximum.

If $\left(\bar{r}_{1}^{e} \bar{r}_{2}^{e}+Q_{12}\right)=0$ and $\left(\sigma_{r 2}^{2}\left(\left(\bar{r}_{1}^{e}\right)^{2}+Q_{11}\right)=\sigma_{r 1}^{2}\left(\left(\bar{r}_{2}^{e}\right)^{2}+Q_{22}\right)\right)$, then an infinitesimal investor is indifferent between any $c_{2 i}$.

Step 2: There are four potential symmetric equilibria.

In any linear symmetric equilibrium all investors choose the same weight in the private signal 
$c_{2 i}=c_{2}$. By substituting the values of $\bar{r}_{1}^{e}$ and $\bar{r}_{2}^{e}$ given by (8) and $Q_{11}, Q_{12}$ and $Q_{22}$ given by (17) into the first order condition given by (23) and imposing that all investors choose the same $c_{2 i}=c_{2}$, I obtain the following expression

$$
\left[-\sigma_{r 2}^{2} \bar{z}_{1} \bar{z}_{2} c_{2}^{2}+\left(\sigma_{r 2}^{2} \sigma_{z 2}^{2}+\sigma_{r 2}^{2} \bar{z}_{2}^{2}-\sigma_{r 1}^{2} \sigma_{z 1}^{2}-\sigma_{r 1}^{2} \bar{z}_{1}^{2}\right) c_{2}+\sigma_{r 1}^{2} \bar{z}_{1} \bar{z}_{2}\right] \frac{2 \Delta}{\left(\sigma_{r 1}^{2}+c_{2}^{2} \sigma_{r 2}^{2}\right)^{2}}=0
$$

where $\Delta$ is a strictly positive expression and it is given by

$$
\Delta=\frac{\sigma_{r 1}^{2} \sigma_{r 2}^{2} \sigma_{z 1}^{2} \sigma_{z 2}^{2}\left(\sigma_{r 1}^{2}+c_{2}^{2} \sigma_{r 2}^{2}\right)}{\rho^{2}\left[\rho^{2}\left(e^{2 \kappa}-1\right)^{2}\left(c_{2}^{2} \sigma_{z 1}^{2}+\sigma_{z 2}^{2}\right)+\sigma_{z 1}^{2} \sigma_{z 2}^{2} e^{2 \kappa}\left(\sigma_{r 1}^{2}+c_{2}^{2} \sigma_{r 2}^{2}\right)\right]}
$$

There are four values of $c_{2}$ that satisfy (25). Thus, there are four potential candidates to a linear symmetric equilibrium given by $c_{2}= \pm \infty, c_{2}=c_{2}^{+}$and $c_{2}=c_{2}^{-}$where

$$
\begin{aligned}
& c_{2}^{+}=\frac{\left(\sigma_{r 2}^{2} \sigma_{z 2}^{2}+\sigma_{r 2}^{2} \bar{z}_{2}^{2}-\sigma_{r 1}^{2} \sigma_{z 1}^{2}-\sigma_{r 1}^{2} \bar{z}_{1}^{2}\right)+\sqrt{\left(\sigma_{r 2}^{2} \sigma_{z 2}^{2}+\sigma_{r 2}^{2} \bar{z}_{2}^{2}-\sigma_{r 1}^{2} \sigma_{z 1}^{2}-\sigma_{r 1}^{2} \bar{z}_{1}^{2}\right)^{2}+4 \sigma_{r 1}^{2} \sigma_{r 2}^{2} \bar{z}_{1}^{2} \bar{z}_{2}^{2}}}{2 \sigma_{r 2}^{2} \bar{z}_{1} \bar{z}_{2}} \\
& \text { and } \\
& c_{2}^{-}=\frac{\left(\sigma_{r 2}^{2} \sigma_{z 2}^{2}+\sigma_{r 2}^{2} \bar{z}_{2}^{2}-\sigma_{r 1}^{2} \sigma_{z 1}^{2}-\sigma_{r 1}^{2} \bar{z}_{1}^{2}\right)-\sqrt{\left(\sigma_{r 2}^{2} \sigma_{z 2}^{2}+\sigma_{r 2}^{2} \bar{z}_{2}^{2}-\sigma_{r 1}^{2} \sigma_{z 1}^{2}-\sigma_{r 1}^{2} \bar{z}_{1}^{2}\right)^{2}+4 \sigma_{r 1}^{2} \sigma_{r 2}^{2} \bar{z}_{1}^{2} \bar{z}_{2}^{2}}}{2 \sigma_{r 2}^{2} \bar{z}_{1} \bar{z}_{2}}
\end{aligned}
$$

In the following steps, I will use the following definitions and expressions. I define $\widehat{\bar{r}}_{1}^{e}$ and $\widehat{\bar{r}}_{2}^{e}$ as the expressions of the expected excess returns given by (8) when all investors choose the same weight of the second asset in the private signal $c_{2 i}=c_{2}$. I also define $\widehat{Q_{11}}, \widehat{Q_{12}}$ and $\widehat{Q_{22}}$ as the expressions for the elements of the variance-covariance matrix of the excess returns given by (17) when all investors choose the same weight of the second asset in the private signal $c_{2 i}=c_{2}$. Finally, the expression for $\left(\widehat{\bar{r}_{1}^{e}} \widehat{\bar{r}_{2}^{e}}+\widehat{Q_{12}}\right)$ is given by

$$
\left(\widehat{\bar{r}}_{1}^{e} \widehat{\bar{r}}_{2}^{e}+\widehat{Q_{12}}\right)=\frac{\sigma_{r 1}^{2} \sigma_{r 2}^{2}}{\rho^{2} \varsigma}\left(\tau_{0}-\tau_{1} c_{2}+\tau_{2} c_{2}^{2}-\tau_{3} c_{2}^{3}+\tau_{4} c_{2}^{4}-\tau_{5} c_{2}^{5}+\tau_{6} c_{2}^{6}-\tau_{7} c_{2}^{7}\right)
$$

where $\tau_{0}, \tau_{1}, \tau_{2}, \tau_{3}, \tau_{4}, \tau_{5}, \tau_{6}, \tau_{7}, \varsigma$ are reported in the additional appendix and are strictly positive expressions. This expression $\left(\widehat{\bar{r}_{1}^{e}} \widehat{\bar{r}_{2}^{e}}+\widehat{Q_{12}}\right)$ depends only on exogenous parameters and $c_{2}$.

Step 3: Eliminate $c_{2}=c_{2}^{-}$and $c_{2}= \pm \infty$ as candidates to a symmetric equilibrium.

I will use the agent's best response function to select a unique candidate, $c_{2}=c_{2}^{+}$, from the set of candidates identified in step 2.

Step 3.1: A symmetric equilibrium where $c_{2}=c_{2}^{-}$given by (27) does not exist.

In a symmetric equilibrium with $c_{2}=c_{2}^{-}$, because $c_{2}^{-}$is strictly negative, the expression $\left(\widehat{\bar{r}}_{1}^{e} \widehat{\bar{r}}{ }_{2}^{e}+\widehat{Q_{12}}\right)$ given by (28) when all investors choose $c_{2}=c_{2}^{-}$is strictly positive. According to step 1.1, this implies that the best response of the infinitesimal investor, $c_{2 i}^{*}$, is given by $(24)$. Because $\left(\widehat{\bar{r}_{1}^{e}} \widehat{\bar{r}_{2}^{e}}+\widehat{Q_{12}}\right)$ evaluated at $c_{2}=c_{2}^{-}$is strictly positive, $c_{2 i}^{*}$ is also strictly positive. This is a contradiction because $c_{2}^{-}$ is a strictly negative expression. In an economy where all investors choose $c_{2}=c_{2}^{-}$, the infinitesimal investor chooses $c_{2 i}^{*} \neq c_{2}^{-}$.

Step 3.2: A symmetric equilibrium where $c_{2}= \pm \infty$ does not exist.

In a symmetric equilibrium with $c_{2}= \pm \infty$, all investors learn only about the second asset. Hence, 
in equilibrium $\pi_{12}=0, \pi_{11}>0, \pi_{22}>0$, where $\pi_{11}, \pi_{12}$ and $\pi_{22}$ are each of the elements in the matrix $\Pi$ given by (15). This implies that the matrices $A_{1}$ and $A_{2}$ in the equilibrium price (16) are diagonal. Hence, in equilibrium $Q_{12}=0$, where $Q_{12}$ is the covariance of the excess returns given by (17) and $\bar{r}_{1}^{e}>0, \bar{r}_{2}^{e}>0$, where $\bar{r}_{1}^{e}$ and $\bar{r}_{2}^{e}$ are the expected excess returns given by (8). Because in equilibrium $\left(\bar{r}_{1}^{e} \bar{r}_{2}^{e}+Q_{12}\right)$ is strictly positive, according to step 1.1, the best response of the infinitesimal investor, $c_{2 i}^{*}$, is given by (24), which is finite. This is a contradiction. In an economy where all investors choose $c_{2}= \pm \infty$, the infinitesimal investor chooses $c_{2 i}^{*} \neq \pm \infty$.

Step 4: If $\bar{r}_{1}^{e+} \bar{r}_{2}^{e+}+Q_{12}^{+} \geq 0$, then there exists a unique linear symmetric rational expectations equilibrium.

I will show that $c_{2}=c_{2}^{+}$is an equilibrium if $\bar{r}_{1}^{e+} \bar{r}_{2}^{e+}+Q_{12}^{+} \geq 0$. According to step $3, c_{2}=c_{2}^{+}$is the only candidate to constitute a linear symmetric rational expectations equilibrium. Hence, if $c_{2}=c_{2}^{+}$ is an equilibrium, then it is a unique linear symmetric equilibrium. Denote by $\left(\bar{r}_{1}^{e+} \bar{r}_{2}^{e+}+Q_{12}^{+}\right)$the expression $\left(\widehat{\bar{r}_{1}^{e}} \widehat{\bar{r}}{ }_{2}^{e}+\widehat{Q_{12}}\right)$ given by (28) evaluated at $c_{2}=c_{2}^{+}$. The sign of expression $\bar{r}_{1}^{e+} \bar{r}_{2}^{e+}+Q_{12}^{+}$ can be positive and negative and it is fully determined by exogenous parameters.

Step 4.1: If $\bar{r}_{1}^{e+} \bar{r}_{2}^{e+}+Q_{12}^{+}>0$, then there exists a unique linear symmetric rational expectations equilibrium.

Assume $\bar{r}_{1}^{e+} \bar{r}_{2}^{e+}+Q_{12}^{+}>0$. In order to prove that $c_{2}=c_{2}^{+}$constitutes a linear symmetric equilibrium, I will show that the best response of an infinitesimal investor when all investors choose $c_{2}=c_{2}^{+}$is given by $c_{2 i}^{*}=c_{2}^{+}$. The first order condition in (23) when all investors choose $c_{2}=c_{2}^{+}$can be rewritten as

$$
\frac{\left[\sigma_{r 1}^{2}\left(\left(\bar{r}_{2}^{e+}\right)^{2}+Q_{22}^{+}\right)-\sigma_{r 2}^{2}\left(\left(\bar{r}_{1}^{e+}\right)^{2}+Q_{11}^{+}\right)\right]}{\left(\bar{r}_{1}^{e+} \bar{r}_{2}^{e+}+Q_{12}^{+}\right)}=\frac{\left(c_{2}^{+2} \sigma_{r 2}^{2}-\sigma_{r 1}^{2}\right)}{c_{2}^{+}}
$$

where $\bar{r}_{1}^{e+}$ and $\bar{r}_{1}^{e+}$ are the expressions of the expected excess returns given by (8) when all investors choose $c_{2 i}=c_{2}^{+}$and $Q_{11}^{+}, Q_{12}^{+}$and $Q_{22}^{+}$are the elements of the variance-covariance matrix of the excess returns given by (17) when all investors choose $c_{2 i}=c_{2}^{+}$. Substituting the RHS of (29) into the best response function given by (24), the revised reaction function if $\bar{r}_{1}^{e+} \bar{r}_{2}^{e+}+Q_{12}^{+}>0$ and all investors choose $c_{2 i}=c_{2}^{+}$can be rewritten as

$$
c_{2 i}^{*}=\frac{\left(c_{2}^{+2} \sigma_{r 2}^{2}-\sigma_{r 1}^{2}\right)}{2 \sigma_{r 2}^{2} c_{2}^{+}}+\sqrt{\left[\frac{\left(c_{2}^{+2} \sigma_{r 2}^{2}-\sigma_{r 1}^{2}\right)}{2 \sigma_{r 2}^{2} c_{2}^{+}}\right]^{2}+\frac{\sigma_{r 1}^{2}}{\sigma_{r 2}^{2}}}
$$

Substituting the expression for $c_{2}^{+}$given by (26) into (30), the revised best response function becomes

$$
c_{2 i}^{*}=\frac{\left(\sigma_{r 2}^{2} \sigma_{z 2}^{2}+\sigma_{r 2}^{2} \bar{z}_{2}^{2}-\sigma_{r 1}^{2} \sigma_{z 1}^{2}-\sigma_{r 1}^{2} \bar{z}_{1}^{2}\right)}{2 \sigma_{r 2}^{2} \bar{z}_{1} \bar{z}_{2}}+\sqrt{\left[\frac{\left(\sigma_{r 2}^{2} \sigma_{z 2}^{2}+\sigma_{r 2}^{2} \bar{z}_{2}^{2}-\sigma_{r 1}^{2} \sigma_{z 1}^{2}-\sigma_{r 1}^{2} \bar{z}_{1}^{2}\right)}{2 \sigma_{r 2}^{2} \bar{z}_{1} \bar{z}_{2}}\right]^{2}+\frac{\sigma_{r 1}^{2}}{\sigma_{r 2}^{2}}}
$$

If $\left(\bar{r}_{1}^{e+} \bar{r}_{2}^{e+}+Q_{12}^{+}\right)>0$, then $c_{2}^{+}$is a fixed point because $c_{2 i}^{*}=c_{2}^{+}$. The optimal precision of the error term in the private signal is given by (21) and in equilibrium the variance of the error term can be characterized as

$$
\Sigma^{+}=\frac{\sigma_{r 1}^{2}+c_{2}^{+2} \sigma_{r 2}^{2}}{\left(e^{2 \kappa}-1\right)}
$$

Step 4.2: If $\bar{r}_{1}^{e+} \bar{r}_{2}^{e+}+Q_{12}^{+}=0$, then there exists a unique linear symmetric rational 
expectations equilibrium.

If $\bar{r}_{1}^{e+} \bar{r}_{2}^{e+}+Q_{12}^{+}=0$, then $\sigma_{r 1}^{2}\left(\left(\bar{r}_{2}^{e+}\right)^{2}+Q_{22}^{+}\right)=\sigma_{r 2}^{2}\left(\left(\bar{r}_{1}^{e+}\right)^{2}+Q_{11}^{+}\right)$because, according to step 2, the first order condition in (23) is always satisfied when all investors choose $c_{2}=c_{2}^{+}$and because $c_{2}^{+}$is strictly positive. Consequently, according to step 1.2, the objective function given by (22) does not depend on $c_{2 i}$ and the infinitesimal investor is indifferent between any attention allocation. Thus, if $\bar{r}_{1}^{e+} \bar{r}_{2}^{e+}+Q_{12}^{+}=0$, then $c_{2}=c_{2}^{+}$is a rational expectations equilibrium. The optimal precision of the error term in the private signal is given by (31).

Step 5: There exists a linear symmetric rational expectations equilibrium only if $\bar{r}_{1}^{e+} \bar{r}_{2}^{e+}+$ $Q_{12}^{+} \geq 0$.

Assume $c_{2}=c_{2}^{+}$is a linear symmetric equilibrium when $\bar{r}_{1}^{e+} \bar{r}_{2}^{e+}+Q_{12}^{+}<0$. Substituting the RHS of the first order condition when all investors choose $c_{2}=c_{2}^{+}$given by (29) into the best response function given by (24), the revised reaction function if $\bar{r}_{1}^{e+} \bar{r}_{2}^{e+}+Q_{12}^{+}<0$ and all investors choose $c_{2 i}=c_{2}^{+}$can be rewritten as

$$
c_{2 i}^{*}=\frac{\left(c_{2}^{2} \sigma_{r 2}^{2}-\sigma_{r 1}^{2}\right)}{2 \sigma_{r 2}^{2} c_{2}}-\sqrt{\left[\frac{\left(c_{2}^{2} \sigma_{r 2}^{2}-\sigma_{r 1}^{2}\right)}{2 \sigma_{r 2}^{2} c_{2}}\right]^{2}+\frac{\sigma_{r 1}^{2}}{\sigma_{r 2}^{2}}}
$$

Substituting the expression for $c_{2}^{+}$given by (26) into (32), the revised reaction function becomes

$$
c_{2 i}^{*}=\frac{\left(\sigma_{r 2}^{2} \sigma_{z 2}^{2}+\sigma_{r 2}^{2} \bar{z}_{2}^{2}-\sigma_{r 1}^{2} \sigma_{z 1}^{2}-\sigma_{r 1}^{2} \bar{z}_{1}^{2}\right)}{2 \sigma_{r 2}^{2} \bar{z}_{1} \bar{z}_{2}}-\sqrt{\left[\frac{\left(\sigma_{r 2}^{2} \sigma_{z 2}^{2}+\sigma_{r 2}^{2} \bar{z}_{2}^{2}-\sigma_{r 1}^{2} \sigma_{z 1}^{2}-\sigma_{r 1}^{2} \bar{z}_{1}^{2}\right)}{2 \sigma_{r 2}^{2} \bar{z}_{1} \bar{z}_{2}}\right]^{2}+\frac{\sigma_{r 1}^{2}}{\sigma_{r 2}^{2}}}
$$

which is a contradiction because $c_{2 i}^{*} \neq c_{2}^{+}$. Thus, if $\bar{r}_{1}^{e+} \bar{r}_{2}^{e+}+Q_{12}^{+}<0$, then a linear symmetric rational expectations equilibrium does not exist.

Hence, there exists a unique linear symmetric rational expectations equilibrium if and only if $\bar{r}_{1}^{e+} \bar{r}_{2}^{e+}+Q_{12}^{+} \geq 0$. In this equilibrium, all investors allocate their information processing capacity to learn about a unique linear combination of asset payoffs where the weight of the second asset is given by (10) and the variance of the error term is given by (11). For expositional purposes, in the main text of the paper, I use $c_{2}=c_{2}^{*}$ to refer to the linear symmetric equilibrium $c_{2}=c_{2}^{+}$.

\subsection{Proof of Proposition 3}

I will show that an equilibrium where all investors specialize in learning does not exist. Proposition 2 shows that a symmetric equilibrium where all investors specialize in learning about one asset does not exist. In what follows, I will show that for any $\lambda$ such that $1>\lambda>0$, there does not exist an asymmetric equilibrium where a fraction $\lambda$ of investors learns only about the first asset, $C_{\lambda}=(1,0)$ and chooses $\Sigma_{\lambda}$ and a fraction $(1-\lambda)$ of investors learns only about the second asset, $C_{1-\lambda}=(1, \infty)$ and chooses $\Sigma_{1-\lambda}$. Assume such an equilibrium exists. Hence, the infinitesimal investor is indifferent between choosing $\left(C_{\lambda}, \Sigma_{\lambda}\right)$ and $\left(C_{1-\lambda}, \Sigma_{1-\lambda}\right)$. The objective function given by (22) provides the same utility to both types of agents when

$$
\frac{\left(\left(\bar{r}_{1}^{e}\right)^{2}+Q_{11}\right)}{\sigma_{r 1}^{2}}=\frac{\left(\left(\bar{r}_{2}^{e}\right)^{2}+Q_{22}\right)}{\sigma_{r 2}^{2}}
$$

Furthermore, in this economy, because investor's private information is about only one asset, in 
equilibrium $\pi_{12}=0, \pi_{11}>0, \pi_{22}>0$ where $\pi_{11}, \pi_{12}$ and $\pi_{22}$ are each of the elements in the matrix $\Pi$ given by (15). This implies that the matrices $A_{1}$ and $A_{2}$ in the equilibrium price (16) are diagonal. Hence, in equilibrium $Q_{12}=0$, where $Q_{12}$ is the covariance of the excess returns given by (17) and $\bar{r}_{1}^{e}>0, \bar{r}_{2}^{e}>0$, where $\bar{r}_{1}^{e}$ and $\bar{r}_{2}^{e}$ are the expected excess returns given by (8). Thus, in equilibrium, $\bar{r}_{1}^{e} \bar{r}_{2}^{e}+Q_{12}>0$ and according to (24), the best response of an infinitesimal investor in this economy is given by

$$
c_{2 i}^{*}=\sqrt{\frac{\sigma_{r 1}^{2}}{\sigma_{r 2}^{2}}}
$$

This leads to a contradiction because the infinitesimal investor wants to deviate from specialization. Therefore, an equilibrium where all investors specialize in learning does not exist.

\subsection{Proof of Proposition 4}

The covariance of asset prices conditional on the asset supplies, $\tilde{Z}$, is given by the element $(1,2)$ of the conditional variance-covariance matrix of asset prices

$$
\operatorname{Var}(\tilde{P} \mid \tilde{Z})=A_{1} \Sigma_{R} A_{1}^{\prime}
$$

where the expression for $A_{1}$ is given in the proof of Proposition 1 in the section 8.1 of the appendix. I define $\pi_{11}^{*}, \pi_{12}^{*}$ and $\pi_{22}^{*}$ as each of the elements in the matrix $\Pi$ given by (15) when all investors choose the same weight of the second asset in the private signal, $c_{2 i}=c_{2}^{*}$, and they can be expressed as

$$
\pi_{11}^{*}=\int_{0}^{1} \rho \Sigma_{i}^{-1} d i=\frac{\rho}{\Sigma^{*}} \quad \pi_{12}^{*}=\int_{0}^{1} \rho \Sigma_{i}^{-1} c_{2 i} d i=\frac{\rho c_{2}^{*}}{\Sigma^{*}} \quad \pi_{22}^{*}=\int_{0}^{1} \rho \Sigma_{i}^{-1} c_{2 i}^{2} d i=\frac{\rho c_{2}^{* 2}}{\Sigma^{*}}
$$

where $c_{2}^{*}$ and $\Sigma^{*}$ are defined in (10) and (11) respectively and are functions of only exogenous parameters. The conditional covariance of the asset prices in the linear symmetric equilibrium, $\operatorname{Cov}^{*}\left(\tilde{P}_{1}, \tilde{P}_{2} \mid \tilde{Z}\right)$, can be expressed as

$$
\operatorname{Cov}^{*}\left(\tilde{P}_{1}, \tilde{P}_{2} \mid \tilde{Z}\right)=\frac{1}{R_{f}^{2}\left[\left(\frac{\rho}{\sigma_{r 1}^{2}}+\rho \Psi\right)\left(\frac{\rho}{\sigma_{r 2}^{2}}+\rho c_{2}^{* 2} \Psi\right)-\rho^{2} c_{2}^{* 2} \Psi^{2}\right]^{2}}\left[\frac{\rho^{2} \pi_{11}^{* 2} c_{2}^{*}\left(\sigma_{z 1}^{2} \sigma_{z 2}^{2}+\rho \pi_{11}^{*} \sigma_{z 2}^{2}+\rho \pi_{11}^{*} c_{2}^{* 2} \sigma_{z 1}^{2}\right)^{2}\left(\sigma_{r 1}^{2}+c_{2}^{* 2} \sigma_{r 2}^{2}\right)}{\sigma_{r 1}^{2} \sigma_{r 2}^{2}\left(\sigma_{z 1}^{2}\right)^{2}\left(\sigma_{z 2}^{2}\right)^{2}}\right]>0
$$

where $\pi_{11}^{*}$ is given by (33) and $\Psi$ is given by

$$
\Psi=\left[\left(\frac{\rho^{2}}{\sigma_{z 1}^{2}}+\frac{\rho^{2} c_{2}^{* 2}}{\sigma_{z 2}^{2}}\right) \frac{1}{\Sigma^{* 2}}+\frac{1}{\Sigma^{*}}\right]
$$

Hence, $\operatorname{Cov}^{*}\left(\tilde{P}_{1}, \tilde{P}_{2} \mid \tilde{Z}\right)$ depends only on exogenous parameters and is a strictly positive expression.

\subsection{Proof of Proposition 5}

The unconditional covariance of asset prices is given by the element $(1,2)$ of the variance-covariance matrix of asset prices

$$
\operatorname{Var}(\tilde{P})=A_{1} \Sigma_{R} A_{1}^{\prime}+A_{2} \Sigma_{Z} A_{2}^{\prime}
$$

where the expressions for $A_{1}$ and $A_{2}$ are given in the proof of Proposition 1 in the section 8.1 of the appendix. The unconditional covariance of asset prices can also be expressed in terms of the 
conditional covariance as $\operatorname{Cov}\left(\tilde{P}_{1}, \tilde{P}_{2}\right)=E\left[\operatorname{Cov}\left(\tilde{P}_{1}, \tilde{P}_{2} \mid \tilde{Z}\right)\right]+\operatorname{Cov}\left(E\left[\tilde{P}_{1} \mid \tilde{Z}\right], E\left[\tilde{P}_{2} \mid \tilde{Z}\right]\right)$. The unconditional covariance of the asset prices in the linear symmetric equilibrium, $\operatorname{Cov}^{*}\left(\tilde{P}_{1}, \tilde{P}_{2}\right)$, can be expressed as

$$
\operatorname{Cov}^{*}\left(\tilde{P}_{1}, \tilde{P}_{2}\right)=\frac{1}{R_{f}^{2}\left[\left(\frac{\rho}{\sigma_{r 1}^{2}}+\rho \Psi\right)\left(\frac{\rho}{\sigma_{r 2}^{2}}+\rho c_{2}^{* 2} \Psi\right)-\rho^{2} c_{2}^{* 2} \Psi^{2}\right]^{2}} \frac{\pi_{11}^{*}}{\sigma_{r 1}^{2} \sigma_{r 2}^{2}\left(\sigma_{z 1}^{2}\right)^{2}\left(\sigma_{z 2}^{2}\right)^{2}}\left(\theta_{1} c_{2}^{*}+\theta_{2} c_{2}^{* 3}+\theta_{3} c_{2}^{* 5}+\theta_{4} c_{2}^{* 7}\right)
$$

where $\Psi$ is given by (34), $\pi_{11}^{*}$ is given by (33) and $\theta_{1}, \theta_{2}, \theta_{3}, \theta_{4}$ are given by

$$
\begin{aligned}
& \theta_{1}=\rho\left(\sigma_{z 1}^{2}\right)^{2}\left(\sigma_{z 2}^{2}\right)^{2}\left[\rho^{2}-\sigma_{r 2}^{2} \sigma_{z 2}^{2}\right]+\rho\left(\sigma_{z 1}^{2}\right)^{2}\left(\sigma_{z 2}^{2}\right)^{2}\left[\rho^{2}-\sigma_{r 1}^{2} \sigma_{z 1}^{2}\right]+\rho^{2} \pi_{11}^{* 3} \sigma_{r 1}^{2}\left(\sigma_{z 2}^{2}\right)^{2}\left[\rho^{2}-\sigma_{r 2}^{2} \sigma_{z 2}^{2}\right]+ \\
& +2 \rho \pi_{11}^{* 2} \sigma_{z 1}^{2} \sigma_{r 1}^{2}\left(\sigma_{z 2}^{2}\right)^{2}\left[\rho^{2}-\sigma_{r 2}^{2} \sigma_{z 2}^{2}\right]+\pi_{11}^{*} \sigma_{z 1}^{2}\left(\sigma_{z 2}^{2}\right)^{2}\left[\rho^{4}-\rho^{2} \sigma_{r 2}^{2} \sigma_{z 2}^{2}-\sigma_{r 1}^{2} \sigma_{z 1}^{2} \sigma_{r 2}^{2} \sigma_{z 2}^{2}\right] \\
& \theta_{2}=2 \rho^{2} \pi_{11}^{* 3} \sigma_{z 1}^{2} \sigma_{z 2}^{2} \sigma_{r 1}^{2}\left[\rho^{2}-\sigma_{r 2}^{2} \sigma_{z 2}^{2}\right]+\rho^{2} \pi_{11}^{* 3} \sigma_{r 2}^{2}\left(\sigma_{z 2}^{2}\right)^{2}\left[\rho^{2}-\sigma_{r 1}^{2} \sigma_{z 1}^{2}\right]+2 \rho \pi_{11}^{* 2} \sigma_{z 2}^{2} \sigma_{r 1}^{2}\left(\sigma_{z 1}^{2}\right)^{2}\left[\rho^{2}-\sigma_{r 2}^{2} \sigma_{z 2}^{2}\right]+ \\
& +2 \rho \pi_{11}^{* 2} \sigma_{z 1}^{2} \sigma_{r 2}^{2}\left(\sigma_{z 2}^{2}\right)^{2}\left[\rho^{2}-\sigma_{r 1}^{2} \sigma_{z 1}^{2}\right]+\pi_{11}^{*} \sigma_{z 2}^{2}\left(\sigma_{z 1}^{2}\right)^{2}\left[\rho^{4}-\rho^{2} \sigma_{r 1}^{2} \sigma_{z 1}^{2}-\sigma_{r 1}^{2} \sigma_{z 1}^{2} \sigma_{r 2}^{2} \sigma_{z 2}^{2}\right] \\
& \theta_{3}=\rho^{2} \pi_{11}^{* 3} \sigma_{r 1}^{2}\left(\sigma_{z 1}^{2}\right)^{2}\left[\rho^{2}-\sigma_{r 2}^{2} \sigma_{z 2}^{2}\right]+2 \rho^{2} \pi_{11}^{* 3} \sigma_{z 1}^{2} \sigma_{z 2}^{2} \sigma_{r 2}^{2}\left[\rho^{2}-\sigma_{r 1}^{2} \sigma_{z 1}^{2}\right]+2 \rho \pi_{11}^{* 2} \sigma_{r 2}^{2}\left(\sigma_{z 1}^{2}\right)^{2} \sigma_{z 2}^{2}\left[\rho^{2}-\sigma_{r 1}^{2} \sigma_{z 1}^{2}\right] \\
& \theta_{4}=\rho^{2} \pi_{11}^{* 3} \sigma_{r 2}^{2}\left(\sigma_{z 1}^{2}\right)^{2}\left[\rho^{2}-\sigma_{r 1}^{2} \sigma_{z 1}^{2}\right]
\end{aligned}
$$

If the following two sufficient conditions are satisfied

$$
\left[\rho^{4}-\rho^{2} \sigma_{r 1}^{2} \sigma_{z 1}^{2}-\sigma_{r 1}^{2} \sigma_{z 1}^{2} \sigma_{r 2}^{2} \sigma_{z 2}^{2}\right] \geq 0
$$

and

$$
\left[\rho^{4}-\rho^{2} \sigma_{r 2}^{2} \sigma_{z 2}^{2}-\sigma_{r 1}^{2} \sigma_{z 1}^{2} \sigma_{r 2}^{2} \sigma_{z 2}^{2}\right] \geq 0
$$

then the unconditional covariance of asset prices is strictly positive because $\theta_{1}, \theta_{2}, \theta_{3}, \theta_{4}$ are strictly positive expressions. These two sufficient conditions can be summarized by a single sufficient condition given by

$$
\min \left\{\left(\rho^{2}-\sigma_{z 1}^{2} \sigma_{r 1}^{2}\right),\left(\rho^{2}-\sigma_{z 2}^{2} \sigma_{r 2}^{2}\right)\right\} \geq \frac{\sigma_{z 1}^{2} \sigma_{r 1}^{2} \sigma_{z 2}^{2} \sigma_{r 2}^{2}}{\rho^{2}}
$$

\subsection{Proof of Proposition 6}

I will show that the relative attention to the second asset in the linear symmetric equilibrium, which is given by $\frac{c_{2}^{* 2} \sigma_{r 2}^{2}}{\sigma_{r 1}^{2}}$, is strictly increasing in its payoff volatility, $\sigma_{r 2}^{2}$, and supply volatility, $\sigma_{z 2}^{2}$. It is enough to show that $\frac{\partial c_{2}^{*}}{\partial \sigma_{r 2}^{2}}>0$ and $\frac{\partial c_{2}^{*}}{\partial \sigma_{z 2}^{2}}>0$. The weight of the second asset in the private signal is strictly increasing in $\sigma_{r 2}^{2}$ since

$$
\frac{\partial c_{2}^{*}}{\partial \sigma_{r 2}^{2}}=\frac{\sigma_{r 1}^{2}}{2 \bar{z}_{1} \bar{z}_{2}\left(\sigma_{r 2}^{2}\right)^{2}}\left[\sigma_{z 1}^{2}+\bar{z}_{1}^{2}+\frac{2\left(\sigma_{z 2}^{2}+\bar{z}_{2}^{2}-\frac{\sigma_{r 1}^{2}}{\sigma_{r 2}^{2}} \sigma_{z 1}^{2}-\frac{\sigma_{r 1}^{2}}{\sigma_{r 2}^{2}} \bar{z}_{1}^{2}\right)\left(\sigma_{z 1}^{2}+\bar{z}_{1}^{2}\right)-4 \bar{z}_{1}^{2} \bar{z}_{2}^{2}}{2 \sqrt{\left(\sigma_{z 2}^{2}+\bar{z}_{2}^{2}-\frac{\sigma_{r 1}^{2}}{\sigma_{r 2}^{2}} \sigma_{z 1}^{2}-\frac{\sigma_{r 1}^{2}}{\sigma_{r 2}^{2}} \bar{z}_{1}^{2}\right)^{2}+4 \frac{\sigma_{r 1}^{2}}{\sigma_{r 2}^{2}} \bar{z}_{1}^{2} \bar{z}_{2}^{2}}}\right]>0
$$

See the additional appendix for additional details on the derivation of this result. The weight of the 
second asset in the private signal is strictly increasing in $\sigma_{z 2}^{2}$ since

$$
\frac{\partial c_{2}^{*}}{\partial \sigma_{z 2}^{2}}=\frac{1}{2 \bar{z}_{1} \bar{z}_{2}}\left[1+\frac{\left(\sigma_{z 2}^{2}+\bar{z}_{2}^{2}-\frac{\sigma_{r 1}^{2}}{\sigma_{r 2}^{2}} \sigma_{z 1}^{2}-\frac{\sigma_{r 1}^{2}}{\sigma_{r 2}^{2}} \bar{z}_{1}^{2}\right)}{\sqrt{\left(\sigma_{z 2}^{2}+\bar{z}_{2}^{2}-\frac{\sigma_{r 1}^{2}}{\sigma_{r 2}^{2}} \sigma_{z 1}^{2}-\frac{\sigma_{r 1}^{2}}{\sigma_{r 2}^{2}} \bar{z}_{1}^{2}\right)^{2}+4 \frac{\sigma_{r 1}^{2}}{\sigma_{r 2}^{2}} \bar{z}_{1}^{2} \bar{z}_{2}^{2}}}\right]>0
$$

Similarly, one can show that the relative attention to the first asset, which is given by $\frac{\sigma_{r 1}^{2}}{c_{2}^{* 2} \sigma_{r 2}^{2}}$, is strictly increasing in its payoff volatility, $\sigma_{r 1}^{2}$, and supply volatility, $\sigma_{z 1}^{2}$.

\subsection{Proof of Proposition 7}

I will show that the posterior variance of the first asset in the linear symmetric equilibrium, $\operatorname{Var}^{*}\left[\tilde{r}_{1} \mid \tilde{Y}_{i}, \tilde{P}\right]$, is increasing in the prior variance of the second asset, $\sigma_{r 2}^{2}$, under a parameter condition. The posterior variance-covariance matrix $V_{i}$ is given by (6) and in equilibrium it can be expressed as

$$
V_{i}^{*}=\left(\begin{array}{cc}
\frac{1}{\sigma_{r 1}^{2}}+\Psi & c_{2}^{*} \Psi \\
c_{2}^{*} \Psi & \frac{1}{\sigma_{r 2}^{2}}+c_{2}^{* 2} \Psi
\end{array}\right)^{-1}
$$

where $\Psi$ is given by (34). The posterior variance of the first asset is given by

$$
\begin{aligned}
\operatorname{Var}^{*}\left[\tilde{r}_{1} \mid \tilde{Y}_{i}, \tilde{P}\right] & =\frac{\frac{1}{\sigma_{r 2}^{2}}+c_{2}^{* 2} \Psi}{\left(\frac{1}{\sigma_{r 1}^{2}}+\Psi\right)\left(\frac{1}{\sigma_{r 2}^{2}}+c_{2}^{* 2} \Psi\right)-c_{2}^{* 2} \Psi^{2}} \\
& =\frac{\sigma_{r 1}^{2}\left(1+c_{2}^{* 2} \sigma_{r 2}^{2} \Psi\right)}{\left(1+c_{2}^{* 2} \sigma_{r 2}^{2} \Psi\right)+\sigma_{r 1}^{2} \Psi} \\
& =\frac{1}{\frac{1}{\sigma_{r 1}^{2}}+\frac{\Psi}{1+c_{2}^{* 2} \sigma_{r, 2}^{2} \Psi}}
\end{aligned}
$$

In order to prove that $\frac{\partial \operatorname{Var}^{*}\left[\tilde{r}_{1} \mid \tilde{Y}_{i}, \tilde{P}\right]}{\partial \sigma_{r 2}^{2}}>0$, it is enough to show that $\frac{\partial\left[\frac{\Psi}{1+c_{2}^{* 2} \sigma_{r, 2}^{2} \Psi}\right]}{\partial \sigma_{r 2}^{2}}<0$, which is given by

$$
\frac{\partial\left[\frac{\Psi}{1+c_{2}^{* 2} \sigma_{r, 2}^{2} \Psi}\right]}{\partial \sigma_{r 2}^{2}}=\frac{\frac{\partial \Psi}{\partial \sigma_{r 2}^{2}}-\Psi^{2}\left(c_{2}^{* 2}+2 c_{2}^{*} \sigma_{r 2}^{2} \frac{\partial c_{2}^{*}}{\partial \sigma_{r 2}^{2}}\right)}{\left(1+c_{2}^{* 2} \sigma_{r, 2}^{2} \Psi\right)^{2}}
$$

where

$$
\frac{\partial \Psi}{\partial \sigma_{r 2}^{2}}=-\frac{2 \rho^{2}\left(\frac{1}{\sigma_{z 1}^{2}}+\frac{c_{2}^{* 2}}{\sigma_{z 2}^{2}}\right) \frac{\partial \Sigma^{*}}{\partial \sigma_{r 2}^{2}}}{\Sigma^{* 3}}+\frac{2 \rho^{2} c_{2}^{*} \frac{\partial c_{2}^{*}}{\partial \sigma_{r 2}^{2}}}{\sigma_{z 2}^{2} \Sigma^{* 2}}-\frac{\frac{\partial \Sigma^{*}}{\partial \sigma_{r 2}^{2}}}{\Sigma^{* 2}}
$$

If $\frac{\partial \Psi}{\partial \sigma_{r 2}^{2}}<0$, then $\frac{\partial\left[\frac{\Psi}{1+c_{2}^{* 2} \sigma_{r, 2}^{2} \Psi}\right]}{\partial \sigma_{r 2}^{2}}<0$. If either(13) or (14) is satisfied, then $\frac{\partial \Psi}{\partial \sigma_{r 2}^{2}}<0$ and $\frac{\partial \operatorname{Var} *\left[\tilde{r}_{1} \mid \tilde{Y}_{i}, \tilde{P}\right]}{\partial \sigma_{r 2}^{2}}>$ 0 . Similarly, one can show that the posterior variance of the second asset is increasing with the prior variance of the first asset if $\sigma_{z 1}^{2} \sigma_{r 1}^{2} \geq \rho^{2}\left(e^{2 \kappa}-1\right)$ or $\sigma_{z 1}^{2} \sigma_{r 1}^{2} \geq \sigma_{z 2}^{2} \sigma_{r 2}^{2}$. 


\subsection{Proof of Proposition 8}

I will show that the expected price of the first asset in the linear symmetric equilibrium, $\bar{p}_{1}^{+}$, is decreasing in the prior variance of the second asset, $\sigma_{r 2}^{2}$, under a parameter condition. Expected asset prices can be written as $\bar{p}_{1}=\left(\bar{r}_{1}-\bar{r}_{1}^{e}\right) / R_{f}$ and $\bar{p}_{2}=\left(\bar{r}_{2}-\bar{r}_{2}^{e}\right) / R_{f}$. The expected excess returns $\bar{R}^{e}=\left(\bar{r}_{1}^{e}, \bar{r}_{2}^{e}\right)^{\prime}$ are given by (8) and in equilibrium they can be expressed as

$$
\bar{R}^{e+}=\left(\begin{array}{cc}
\frac{\rho}{\sigma_{r 1}^{2}}+\frac{\rho \pi_{11}^{* 2}}{\sigma_{z 1}^{2}}+\frac{\rho \pi_{12}^{* 2}}{\sigma_{z 2}^{2}}+\pi_{11}^{*} & \frac{\rho \pi_{11}^{*} \pi_{12}^{*}}{\sigma_{z 1}^{2}}+\frac{\rho \pi_{12}^{*} \pi_{22}^{*}}{\sigma_{z 2}^{2}}+\pi_{12}^{*} \\
\frac{\rho \pi_{11}^{*} \pi_{12}^{*}}{\sigma_{z 1}^{2}}+\frac{\rho \pi_{12}^{*} \pi_{22}^{*}}{\sigma_{z 2}^{2}}+\pi_{12}^{*} & \frac{\rho}{\sigma_{r 2}^{2}}+\frac{\rho \pi_{12}^{* 2}}{\sigma_{z 1}^{2}}+\frac{\rho \pi_{22}^{* 2}}{\sigma_{z 2}^{2}}+\pi_{22}^{*}
\end{array}\right)^{-1} \bar{Z}
$$

where expressions for $\pi_{11}^{*}, \pi_{12}^{*}$ and $\pi_{22}^{*}$ are given by (33). The expected excess return of the first asset is given by

$$
\begin{aligned}
\bar{r}_{1}^{e+} & =\frac{\rho\left(\frac{1}{\sigma_{r 2}^{2}}+c_{2}^{* 2} \Psi\right) \bar{z}_{1}-\rho c_{2}^{*} \Psi \bar{z}_{2}}{\rho^{2}\left(\frac{1}{\sigma_{r 1}^{2}}+\Psi\right)\left(\frac{1}{\sigma_{r 2}^{2}}+c_{2}^{* 2} \Psi\right)-\rho^{2} c_{2}^{* 2} \Psi^{2}} \\
& =\frac{\sigma_{r 1}^{2}}{\rho}\left[\frac{\bar{z}_{1}+c_{2}^{*} \sigma_{r 2}^{2}\left(c_{2}^{*} \bar{z}_{1}-\bar{z}_{2}\right) \Psi}{1+\left(\sigma_{r 1}^{2}+c_{2}^{* 2} \sigma_{r 2}^{2}\right) \Psi}\right]
\end{aligned}
$$

where $\Psi$ is defined in (34). In order to prove that $\frac{\partial \bar{p}_{1}^{+}}{\partial \sigma_{r 2}^{2}}<0$, it is enough to show that $\frac{\partial \bar{r}_{1}^{e+}}{\partial \sigma_{r 2}^{2}}>0$, which is given by

$$
\frac{\partial \bar{r}_{1}^{e+}}{\partial \sigma_{r 2}^{2}}=D_{0}\left(D_{1}+D_{2}\right)
$$

where

$$
\begin{aligned}
D_{0} & =\frac{\sigma_{r 1}^{2}}{\rho} \frac{1}{\left[1+\left(\sigma_{r 1}^{2}+c_{2}^{* 2} \sigma_{r 2}^{2}\right) \Psi\right]^{2}} \\
D_{1} & =-\left(c_{2}^{*} \bar{z}_{2}+\sigma_{r 2}^{2} \frac{\partial c_{2}^{*}}{\sigma_{r 2}^{2}} \bar{z}_{2}\right) \Psi-\left(\sigma_{r 1}^{2} \bar{z}_{1}+c_{2}^{*} \sigma_{r 2}^{2} \bar{z}_{2}\right) \frac{\partial \Psi}{\partial \sigma_{r 2}^{2}} \\
D_{2} & =\left[\sigma_{r 1}^{2} c_{2}^{*}\left(c_{2}^{*} \bar{z}_{1}-\bar{z}_{2}\right)+\sigma_{r 1}^{2} \sigma_{r 2}^{2} \frac{\partial c_{2}^{*}}{\sigma_{r 2}^{2}}\left(c_{2}^{*} \bar{z}_{1}-\bar{z}_{2}\right)+c_{2}^{*} \sigma_{r 2}^{2} \frac{\partial c_{2}^{*}}{\sigma_{r 2}^{2}}\left(\sigma_{r 1}^{2} \bar{z}_{1}+c_{2}^{*} \sigma_{r 2}^{2} \bar{z}_{2}\right)\right] \Psi^{2}
\end{aligned}
$$

The expression for $D_{0}$ given by (36) is strictly positive. If $\sigma_{z 2}^{2} \sigma_{r 2}^{2} \geq \sigma_{z 1}^{2} \sigma_{r 1}^{2}$, then $D_{1}>0$ and $D_{2}>0$. Hence, if $\sigma_{z 2}^{2} \sigma_{r 2}^{2} \geq \sigma_{z 1}^{2} \sigma_{r 1}^{2}$, then $\frac{\partial \bar{r}_{1}^{e+}}{\partial \sigma_{r 2}^{2}}>0$ and $\frac{\partial \bar{p}_{1}^{+}}{\partial \sigma_{r 2}^{2}}<0$. Similarly, one can show that the expected price of the second asset is decreasing in the prior variance of the first asset if $\sigma_{z 2}^{2} \sigma_{r 2}^{2} \leq \sigma_{z 1}^{2} \sigma_{r 1}^{2}$.

\section{References}

[1] A. Admati, A Noisy Rational Expectations Equilibrium for Multi-Asset Securities Markets, Econometrica 53 (1985), 629-657.

[2] N. Barberis, A. Shleifer, J. Wurgler, Comovement, Journal of Financial Economics 75 (2005), 283-318. 
[3] G. Calvo, Contagion in Emerging Markets: when Wall Street is a Carrier, Working Paper, 1999.

[4] S. Corwin, J. Coughenour, Limited Attention and the Allocation of Effort in Securities Trading, Journal of Finance 63 (2008), 3031-3067.

[5] R. Greenwood, Excess Comovement of Stock Returns: Evidence from Cross-sectional Variation in Nikkei 225 Weights, Review of Financial Studies 21 (2008), 1153-1186.

[6] A. Hameed, R. Morck, J. Shen, B. Yeung, Information Markets, Analysts, and Comovement in Stock Returns, NYU Working Paper, 2008.

[7] C. Hellwig, L. Veldkamp, Knowing What Others Know: Coordination Motives in Information Acquisition, Review of Economic Studies 76 (2009), 223-251.

[8] J. Kallberg, P. Pasquariello, Time-Series and Cross-Sectional Excess Comovement in Stock Indexes, Journal of Empirical Finance 15 (2008), 481-502.

[9] A. Khinchin, Mathematical Foundations of Information Theory, Dover Publications, Inc., New York, 1957.

[10] M. King, S. Wadhwani, Transmission of Volatility between Stock Markets, Review of Financial Studies 3 (1990), 5-33.

[11] D. Kreps, E. Porteus, Temporal Resolution of Uncertainty and Dynamic Choice Theory, Econometrica 46 (1978), 185-200.

[12] D. Kreps, E. Porteus, Temporal von Neumann-Morgenstern and Induced Preferences, Journal of Economic Theory 20 (1979), 81-109.

[13] L. Kodres, M. Pritsker, A Rational Expectations Model of Financial Contagion, Journal of Finance 57 (2002), 769-799.

[14] A. Kyle, W. Xiong, Contagion as a Wealth Effect, Journal of Finance 56 (2001), 14011440 .

[15] Y. Luo, Consumption Dynamics under Information Processing Constraints, Review of Economic Dynamics 11 (2008), 366-385.

[16] B. Mackowiak, M. Wiederholt, Optimal Sticky Prices under Rational Inattention, The American Economic Review 99 (2009), 769-803.

[17] B. Mackowiak, M. Wiederholt, Business Cycle Dynamics under Rational Inattention, Working Paper, 2009.

[18] P. Pasquariello, C. Vega, Strategic Trading in the U.S. Stock Market, Working Paper, 2009.

[19] L. Peng, Learning with Information Capacity Constraints, Journal of Financial and Quantitative Analysis 40 (2005), 307-329. 
[20] L. Peng, W. Xiong, Investor Attention, Overconfidence and Category Learning, Journal of Financial Economics 80 (2006), 563-602.

[21] R. Pindyck, J. Rotemberg, The Comovement of Stock Prices, Quarterly Journal of Economics 108 (1993), 1073-1104.

[22] C. Sims, Implications of Rational Inattention, Journal of Monetary Economics 50 (2003), 665-690.

[23] C. Sims, Rational Inattention: A Research Agenda, The American Economic Review 96 (2006), 158-163.

[24] M. Spence, R. Zeckhauser, The Effect of the Timing of Consumption Decisions and the Resolution of Lotteries on the Choice of Lotteries, Econometrica 40 (1972), 401-403.

[25] S. Van Nieuwerburgh, L. Veldkamp, Information Acquisition and Portfolio UnderDiversification, Forthcoming in the Review of Economic Studies, 2009.

[26] S. Van Nieuwerburgh, L. Veldkamp, Information Immobility and the Home Bias Puzzle, Journal of Finance 64 (2009), 1187-1215.

[27] L. Veldkamp, Information Markets and the Comovement of Asset Prices, Review of Economic Studies 73 (2006), 823-845.

[28] R. Verrecchia, Information Acquisition in a Noisy Rational Expectations Economy, The American Economic Review 50 (1982), 1415-1430.

[29] K. Yuan, Asymmetric Price Movements and Borrowing Constraints: A REE Model of Crisis, Contagion, and Confusion, The Journal of Finance 60 (2005), 379-411. 


\section{$9 \quad$ Figures}
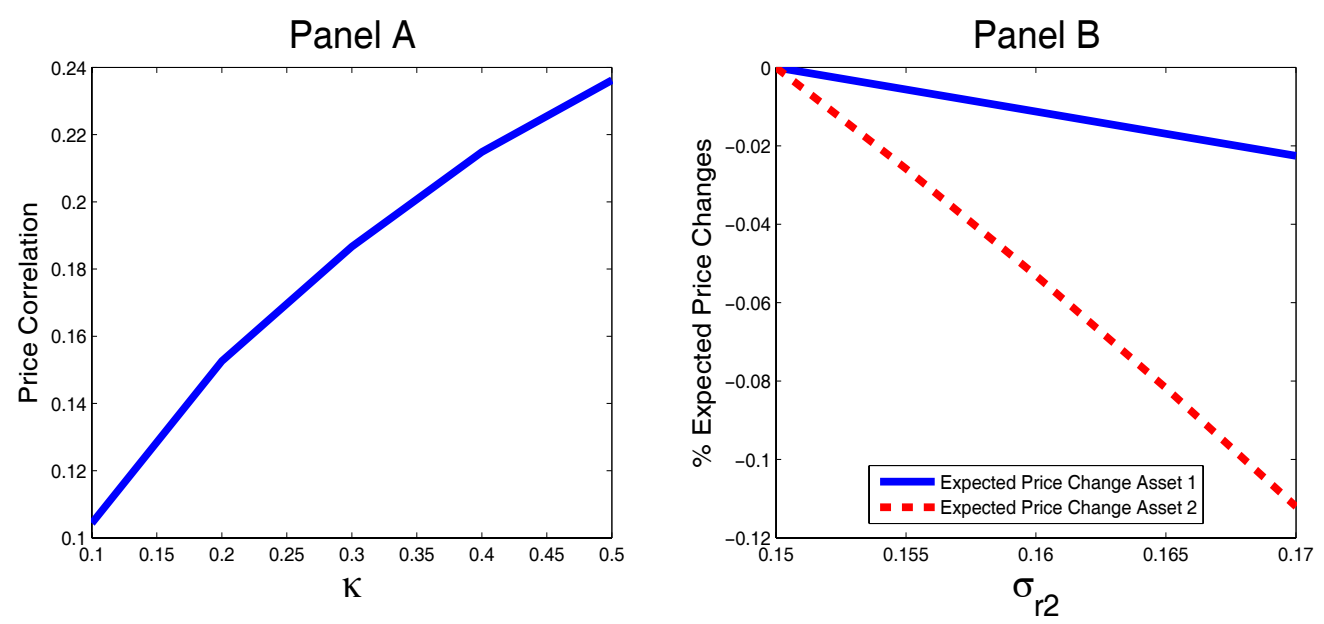

Figure1: Panel A shows the price correlation of the first and second asset for several values of information processing capacity, $\kappa$. Panel B shows the percentage change of expected asset prices for several values of the standard deviation of the second asset with respect to the benchmark $\left(\sigma_{r 2}=0.15\right)$. The parameter values are the following $\sigma_{r 1}=0.15, \bar{r}_{1}=\bar{r}_{2}=1, \sigma_{z 1}=\sigma_{z 2}=10, \bar{z}_{1}=\bar{z}_{2}=100, \rho=2, \kappa=0.4$. 